\title{
NEW APPROACH IN THE THEORY OF STELLAR EQUILIBRIUM WITH AXIAL ROTATION
}

\author{
M. V. Vavrukh(D, N. L. Tyshko(D), D. V. Dzikovskyi (D) \\ Ivan Franko National University of Lviv, Department for Astrophysics, \\ 8, Kyrylo and Methodiy St., Lviv, UA-79005, Ukraine
}

(Received 06 May 2020; in final form 23 May 2020; accepted 27 May 2020; published online 22 September 2020)

\begin{abstract}
The study proposes a new method for the calculation of the equilibrium configuration of polytropes with rigid-body axial rotation. Self-consistency is based on the simultaneous use of differential and integral forms of the mechanical equilibrium equation and a new variant of the perturbation theory, which is relative to the rotation influence. The solutions are shown in the form of expansions for the Legendre polynomials and the functions of radial coordinate. The integral form of the equilibrium equation allows us to correctly define the set of the integration constants (the expansion coefficients, which depend on the angular velocity). The geometrical parameters of the stellar surface as well as the mass, volume and the moment of inertia were calculated as the functions of the angular velocity at fixed values of the polytropic index $n=0 ; 1.0 ; 1.5 ; 2.0 ; 2.5 ; 3.0$. A comparison with the results of other authors was performed.
\end{abstract}

Key words: polytropic stars, heterogeneous ellipsoids, axial rotation, mechanical equilibrium equation, stability of stars.

DOI: https://doi.org/10.30970/jps.24.3902

\section{INTRODUCTION}

The fundamentals of the polytropic theory of Solarlike stars were laid down by the works of H. Lane [1], K. Emden [2], R. Fowler [3], A. Eddington [4] and other researchers in the first half of the last century. This theory is based on the equilibrium equation of star with the polytropic equation of state:

$$
P(\mathbf{r})=K \rho^{\nu}(\mathbf{r}) \equiv K \rho^{1+1 / n}(\mathbf{r}),
$$

where $P(\mathbf{r})$ is the pressure in point with radius-vector $\mathbf{r}, \rho(\mathbf{r})$ is the local density of matter, $K$ and $\nu$ are the constants. It yields the determination of the main relations between the polytrope characteristics and describes their stability. The idea of the polytropic dependence between the pressure and density was successfully used for the construction of the cold degenerate dwarfs theory [5].

Axial rotation is the factor, which is inherent in the various celestial objects - the stars of main sequence, pulsars, white dwarfs and black holes. The equilibrium equation of polytrope without rotation with spherical symmetry of matter distribution is an ordinary differential equation of second order, which is known as the LaneEmden-Fowler equation. The equilibrium equation for the polytropic model with axial rotation in the general case (at any value of the index $n$ ) is a non-linear differential equation of second order in partial derivatives. Only in the particular case $n=0$ the exact solution of the equation is known, from which the Maclaurin formula (see [6]) is obtained, that determines the relations between the angular velocity and the eccentricity of the rotational homogeneous ellipsoid.

To evaluate the influence of rotation on the solar characteristics, E. Milne [7] found the approximate solution of the equilibrium equation at $n=3$ for the case of small angular velocity, by linearizing the equati- on. Such approximation corresponds to the first order of perturbation theory. Using the method of E. Milne [7], S. Chandrasekhar [8] obtained the solutions for the polytropes with indexes $n=1.0,1.5,2.0,2.5,3.0$ with the help of the numerical integration. Z. Kopal [9] remarked that in the partial case $n=1$ with axial symmetry in the distribution of matter, the equilibrium equation allows the separation of variables. He found the set of fundamental solutions in the form of products of Legendre polynomials and the spherical Bessel function of the first kind. However, Z. Kopal did not consider the question of finding of general solution, by the given boundary conditions.

R. James [10] went beyond the small rotational velocity approximation. He found an approximate solution for the polytropes with indexes $n=1.0,1.5,2.0,2.5,3.0$ and calculated the dependence of the polytrope characteristics on the angular velocity in the interval $0 \leq \omega \leq$ $\omega_{\max }(n)$. Unfortunately, the solutions were not present in the publication, which makes it impossible to analyze their dependence on the angular velocity or the solutions for the calculation of other characteristics.

The work [11] generalizes the E. MilneS. Chandrasekhar approach by a more accurate description of the outer polytrope region. Aiming to find the integration constants in the works $[7,8]$ and also to determine the fitting parameters in the work [11], authors applied the approximation traditional in the stellar surface theory, which is based on the usage of the general multipole form of potential, created by the unknown distribution of matter in the inner part of the star. The common approximation of these works is the first approximation relative to rotation influence. Therefore the integration constants and fitting parameters do not depend on the angular velocity, but only on the polytropic index $[7,8,11]$. The partial solution of the equilibrium equation which is considered in the work [12] at $n=1$ improves the 
S. Chandrasekhar's solution [8] by the determination of the integration constants numerically.

In spite of the long research history, the problem of the calculation of characteristics of the polytropic model remains relevant and has both methodological and applied importance. Recent works [13, 14] should be mentioned, which use computer methods to calculate the characteristics of specific stars based on the polytropic model at $n=1$. The polytrope model is a good zero approximation for the calculation of the characteristics of massive dwarfs $[10,15]$. It can be used to describe neutron stars, circumstellar disks, gas giant planets, in the theory of stability and pulsation of stars.

The work [16] showed that to find more accurate solutions of the equilibrium equation for the polytropes with rotation it is necessary to use the multi-component expansion for the Legendre polynomials. In this case, the correct definition of the integration constants is provided by the integral form of the equilibrium equation, which is equivalent to the explicit calculation of the gravitational potential at this point of polytrope by the known solution of the equilibrium equation.

Of course, the solutions of the equilibrium equation in the presence of rotation are found by the perturbation theory, and in the role of zero approximation the K. Emden's function $y_{n}(\xi)$ is used. It takes the negative values beyond the K. Emden radius and therefore cannot be the optimal approximation. This problem can be omitted by searching the approximate solution of the equilibrium equation in the outer region (periphery), as it was done in [11]. In this work we proposed another way. We use a single solution form for the whole polytrope. But in the role of zero approximation we choose the model of monotonically decreasing function of the radial coordinate, which is positive inside the polytrope. It allows the correct linearization of the equation at $n \neq 1$. It also improves the convergence of the series for the Legendre polynomials at arbitrary values $n$, as evidenced by the calculations of integration constants, which depend on the angular velocity.

The purpose of our work is to obtain solutions of mechanical equilibrium for the polytropes with axial rotation, which will allow us in the future to build correct polytropic models for real stars of different types (stars of the main sequence, white dwarfs, neutron stars) based on observed data. We have calculated the dependence of the polar and equatorial radii, eccentricity, mass and moment of inertia of polytropes with indexes $n=1.0,1.5,2.0,2.5,3.0$ on the rotational velocity. The comparison was performed with the results of the critical parameters calculations in the works $[8,10,11]$.

\section{GENERAL RELATIONS}

In the general case (in the presence of rotation), the equilibrium equation is rewritten in a non-inertial (rotating) coordinate system in the form [8]

$$
\nabla P(\mathbf{r})=-\rho(\mathbf{r})\left\{\nabla \Phi_{\text {grav }}(\mathbf{r})+\nabla \Phi_{c}(\mathbf{r})\right\},
$$

where

$$
\Phi_{\text {grav }}(\mathbf{r})=-G \int \frac{d \mathbf{r}^{\prime} \rho\left(\mathbf{r}^{\prime}\right)}{\left|\mathbf{r}-\mathbf{r}^{\prime}\right|}
$$

is the gravitational potential inside the star and $\Phi_{c}(\mathbf{r})$ is the centrifugal potential. If the axis $O z$ of the spherical coordinate system coincides with the axis of rotation then

$$
\Phi_{c}(\mathbf{r})=-\frac{1}{2} \omega^{2} r^{2} \sin ^{2} \theta
$$

Here $\theta$ is the polar angle, $\omega$ is the angular velocity of the reference frame, which is considered to be constant.

Substituting expressions (1), (3) and (4) in equation (2) and using the identity

$$
\left(1+\frac{1}{n}\right) \rho^{1 / n-1}(\mathbf{r}) \nabla \rho(\mathbf{r})=(1+n) \nabla \rho^{1 / n}(\mathbf{r}),
$$

the equilibrium equation is obtained in the form of a differential equation that determines the density distribution,

$$
K(1+n) \Delta \rho^{1 / n}(\mathbf{r})=-4 \pi G \rho(\mathbf{r})+\frac{1}{2} \omega^{2} \Delta\left(r^{2} \sin ^{2} \theta\right) .
$$

In the presence of axial symmetry $(\rho(\mathbf{r})=\rho(r, \theta))$ the Laplace operator is written in the form

$$
\begin{aligned}
& \Delta=\Delta_{r}+\frac{1}{r^{2}} \Delta_{\theta}, \quad \Delta_{r}=\frac{1}{r^{2}} \frac{\partial}{\partial r}\left(r^{2} \frac{\partial}{\partial r}\right), \\
& \Delta_{\theta}=\frac{\partial}{\partial t}\left(1-t^{2}\right) \frac{\partial}{\partial t}
\end{aligned}
$$

at $t=\cos \theta$, therefore $\Delta\left(r^{2} \sin ^{2} \theta\right)=4$. Introducing the dimensionless radial coordinate $\xi=r / \lambda_{n}$, as well as using the substitution

$$
\rho(r, \theta)=\rho_{c} Y^{n}(\xi, \theta),
$$

where $\rho_{c}$ is the density of matter in the stellar center, we transform equation (6) to a dimensionless form

$$
\Delta(\xi, \theta) Y(\xi, \theta)=\Omega^{2}-Y^{n}(\xi, \theta) .
$$

Here the scale $\lambda_{n}$, dimensionless angular velocity $\Omega$ and Laplacian are determined by the relations

$$
\begin{aligned}
& K(1+n)=4 \pi G \lambda_{n}^{2} \rho_{c}^{1-1 / n}, \Omega^{2}=\omega^{2}\left(2 \pi G \rho_{c}\right)^{-1}, \\
& \Delta(\xi, \theta)=\Delta_{\xi}+\frac{1}{\xi^{2}} \Delta_{\theta}, \Delta_{\xi}=\frac{1}{\xi^{2}} \frac{\partial}{\partial \xi}\left(\xi^{2} \frac{\partial}{\partial \xi}\right) .
\end{aligned}
$$

According to the definition (8), $Y(0, \theta)=1$ and the condition $\partial Y(\xi, \theta) / \partial \xi=0$ at $\xi=0$ corresponds to the solutions regular in the vicinity $\xi=0$. At large values of $\Omega$ the non-monotonic dependence $Y(\xi, \theta)$ on the variable 
$\xi$ in the equator region as well as leakage of matter are possible. The stability conditions of stars in the equator region

$$
Y\left(\xi, \frac{\pi}{2}\right)=0, \quad \frac{\partial}{\partial \xi} Y\left(\xi, \frac{\pi}{2}\right)=0
$$

determine the maximal permissible value of the parameter $\Omega_{\max }^{(n)}$ and the corresponding value of the equatorial radius $\xi_{e}^{\max }(n)$. According to the definition $(8)$, only positive solutions of equation (9) have physical meaning, which is a two-dimensional differential equation of the second order in partial derivatives with two dimensionless parameters $n, \Omega \geq 0$.

Equation (9) is similar to the Poisson equation, therefore it can formally be considered as an equation for the dimensionless gravitational potential, which is created by the dimensionless density distribution $(4 \pi)^{-1} \cdot\left\{\Omega^{2}-Y^{n}(\xi, \theta)\right\}$.

In this regard, this equation can be rewritten in the integral form

$$
\begin{aligned}
Y(\xi, \theta) & =1+\sum_{l=1}^{\infty} C_{2 l} \xi^{2 l} P_{2 l}(t) \\
& -\frac{1}{4 \pi} \int\left\{\Omega^{2}-Y^{n}\left(\xi^{\prime}, \theta^{\prime}\right)\right\} Q\left(\boldsymbol{\xi}, \boldsymbol{\xi}^{\prime}\right) d \boldsymbol{\xi}^{\prime}
\end{aligned}
$$

where $C_{2 l}$ are the integration constants, $P_{2 l}(t)$ is the Legendre's polynomials of the $2 l$-th order, the kernel of the equation is

$$
Q\left(\boldsymbol{\xi}, \boldsymbol{\xi}^{\prime}\right)=\left\{\left|\boldsymbol{\xi}-\boldsymbol{\xi}^{\prime}\right|^{-1}-\left(\xi^{\prime}\right)^{-1}\right\},
$$

and the integration is performed over the stellar volume. Taking into account the identity

$$
\Delta(\xi, \theta)\left\{\xi^{l} P_{l}(t)\right\}=0,
$$

it is easy to verify that equations (9) and (12) are equivalent.

The root of equation $Y\left(\xi_{0}(\theta), \theta\right)=0$ determines the equation of the second-order curve (which is close to the ellipse), whose rotation relative to the axis $O z$ forms the stellar surface. The expressions for the mass, volume and moment of inertia of a star are as follows:

$$
\begin{aligned}
& M(n, \Omega)=2 \pi \lambda_{n}^{3} \rho_{c} \int_{0}^{\pi} \sin \theta d \theta \int_{0}^{\xi_{0}(\theta)} \xi^{2} Y^{n}(\xi, \theta) d \xi \\
& V(n, \Omega)=\frac{2 \pi}{3} \lambda_{n}^{3} \int_{0}^{\pi} \sin \theta \xi_{0}^{3}(\theta) d \theta \\
& I(n, \Omega)=4 \pi \lambda_{n}^{5} \rho_{c} \int_{0}^{\pi} \sin ^{3} \theta d \theta \int_{0}^{\xi_{0}(\theta)} \xi^{4} Y^{n}(\xi, \theta) d \xi .
\end{aligned}
$$

At the arbitrary value of the polytropic index $n$ the gravitational potential inside a star (3) is related to the dimensionless potential

$$
\Phi_{n}(\boldsymbol{\xi})=-\frac{1}{4 \pi} \int \frac{Y^{n}\left(\boldsymbol{\xi}^{\prime}\right)}{\left|\boldsymbol{\xi}-\boldsymbol{\xi}^{\prime}\right|} d \boldsymbol{\xi}^{\prime}
$$

with such expression

$$
\Phi_{\text {grav }}(\mathbf{r})=4 \pi G \lambda_{n}^{2} \rho_{c} \Phi_{n}(\boldsymbol{\xi}) .
$$

Rewriting equation (2) in dimensionless variables, we obtain the relation

$$
\frac{\partial}{\partial \xi}\left\{\Phi_{n}(\xi, \theta)+Y(\xi, \theta)\right\}=\frac{\Omega^{2}}{3} \xi\left\{1-P_{2}(t)\right\} .
$$

Equation (12) can be represented in the terms $Y(\xi, \theta)$, $\Phi_{n}(\xi, \theta)$, namely

$$
\begin{aligned}
& Y(\xi, \theta)+\left\{\Phi_{n}(\xi, \theta)-\Phi_{n}(0,0)\right\} \\
& =1+\sum_{l=1} C_{2 l} \xi^{2 l} P_{2 l}(t)+\Omega^{2}\left\{\Phi_{0}(\xi, \theta)-\Phi_{0}(0,0)\right\}
\end{aligned}
$$

where $\Phi_{0}(\xi, \theta)$ is the dimensionless model potential with the constant density $\rho_{0}=1$. This allows us to convert equation (18) to the following form

$$
\begin{aligned}
& \frac{\partial}{\partial \xi}\left\{\sum_{l=1} C_{2 l} \xi^{2 l} P_{2 l}(t)+\Omega^{2}\left[\Phi_{0}(\xi, \theta)-\Phi_{0}(0,0)\right]\right\} \\
& =\xi \frac{\Omega^{2}}{3}\left(1-P_{2}(t)\right) .
\end{aligned}
$$

The difference of potentials $\Phi_{0}(\xi, \theta)-\Phi_{0}(0,0)$ is easy to calculate using expansion in the series of kernel $Q\left(\boldsymbol{\xi}, \boldsymbol{\xi}^{\prime}\right)$ for the Legendre polynomials and performing integration over the variable $\boldsymbol{\xi}^{\prime}$ within the rotational ellipsoid with eccentricity $e$ and equatorial radius $\xi_{e}$, which we will define in a self-consistent manner later. In this way we find that

$$
\begin{aligned}
& \Phi_{0}(\xi, \theta)-\Phi_{0}(0,0)=-\frac{1}{4 \pi} \int d \boldsymbol{\xi}^{\prime} Q\left(\boldsymbol{\xi}, \boldsymbol{\xi}^{\prime}\right) \\
& =\frac{\xi^{2}}{6}-\frac{\xi^{2}}{2} P_{2}(t) \int_{-1}^{+1} d t^{\prime} P_{2}\left(t^{\prime}\right) \int_{\xi}^{\xi_{0}\left(t^{\prime}\right)} \frac{d \xi^{\prime}}{\xi^{\prime}},
\end{aligned}
$$

where

$$
\xi_{0}\left(t^{\prime}\right)=\xi_{e}\left\{1+\left(t^{\prime}\right)^{2} \frac{e^{2}}{1-e^{2}}\right\}^{-1 / 2}
$$

By integrating over the variables $\xi^{\prime}$ and $t^{\prime}$ we obtain the final expression (see [18]):

$$
\begin{aligned}
& \Phi_{0}(\xi, \theta)-\Phi_{0}(0,0)=\frac{\xi^{2}}{6}+\frac{\xi^{2}}{2} P_{2}(t) I(e), \\
& I(e)=\frac{2}{3}+\frac{1-e^{2}}{e^{2}}-\frac{\left(1-e^{2}\right)^{1 / 2}}{e^{3}} \arcsin e
\end{aligned}
$$

Varying the eccentricity in the limits $0 \leq e \leq 1$ the function $I(e)$ changes in the region $2 e^{2} / 15 \leq I(e) \leq 2 / 3$. Substituting expression (23) in equation (20) we get the equality

$$
\sum_{l=1} C_{2 l} 2 l \xi^{2 l-2} P_{2 l}(t)=-\frac{\Omega^{2}}{3} P_{2}(t)(1+3 I(e)) .
$$


According to the orthogonality of the Legendre polynomials, it follows that

$$
C_{2}=-\frac{\Omega^{2}}{6}(1+3 I(e)),
$$

and all other constants $C_{2 l}=0$.

Therefore, equation (12) for arbitrary values of the polytropic index $n$ can be written in the form

$$
\begin{aligned}
Y(\xi, \theta) & =1+\frac{\Omega^{2} \xi^{2}}{6}\left(1-P_{2}(t)\right) \\
& +\frac{1}{4 \pi} \int Y^{n}\left(\xi^{\prime}, \theta^{\prime}\right) Q\left(\boldsymbol{\xi}, \boldsymbol{\xi}^{\prime}\right) d \boldsymbol{\xi}^{\prime}
\end{aligned}
$$

Equations (9) and (26) are a closed system, which does not require any additional information to determine the general solution $Y(\xi, \theta)$ that correspond to the given boundary conditions. We solve this system selfconsistently, thus we achieve a correct description of the polytrope surface, in contrast to the works [7, 8, 11], which used an approximate description of the periphery region.

\section{THE EMDEN EQUATION}

The partial case $\Omega=0$ corresponds to the polytropic star with spherical symmetry and the equation (9) becomes the one-dimensional one-parametric equation [17]

$$
\Delta_{\xi} y(\xi)=-y^{n}(\xi)
$$

with the boundary conditions $y(0)=1, d y / d \xi=0$ at $\xi=0$, as well as the physical condition $y(\xi) \geq 0$. These solutions at fixed $n$ are used in the role of the zero approximation in equation (9). The dimensionless stellar radius $\xi_{1}$ is a smaller root of the equation $y(\xi)=0$. All stellar characteristics are determined by the polytropic index $n \geq 0$. The value $n=5$ is critical: at $n<5$ solutions of equation (27) are alternating-sign functions of $\xi$, and at $n \geq 5$ they are positive in the whole range $0 \leq \xi \leq \infty$ and the boundary condition on the stellar surface is not fulfilled $\left(\xi_{1}(5)=\infty\right.$, that corresponds to a star without an external border). Henceforward we only consider the models with $n \leq 5(\nu \geq 6 / 5)$. After integration over kernel angular variables $Q\left(\boldsymbol{\xi}, \boldsymbol{\xi}^{\prime}\right)$, equation (12) takes the form

$$
y(\xi)=1+\int_{0}^{\xi}\left\{\frac{\left(\xi^{\prime}\right)^{2}}{\xi}-\xi^{\prime}\right\} y^{n}\left(\xi^{\prime}\right) d \xi^{\prime} .
$$

The characteristic features of solutions of equations (27), (28) are well known [17]. They are illustrated by the accurate analytical solutions at $n=0,1,5$, namely

$$
\begin{aligned}
& y_{0}(\xi)=1-\xi^{2} / 6 ; \xi_{1}(0)=\sqrt{6} ; \\
& y_{1}(\xi)=\frac{1}{\xi} \sin \xi ; \xi_{1}(1)=\pi ; \\
& y_{5}(\xi)=\left\{1+\xi^{2} / 3\right\}^{-1 / 2} ; \xi_{1}(5)=\infty .
\end{aligned}
$$

For other values of index $n$, we can get the solution through the numerical integration of equation (27) or (28). In the range of small values $\xi$, the Emden functions have the following series expansion [17]:

$$
y_{n}(\xi)=1-\frac{1}{3 !} \xi^{2}+\frac{n}{5 !} \xi^{4}-\frac{n(8 n-5)}{3 \cdot 7 !} \xi^{6}+\ldots
$$

In the case of $n>1$, equation (27) can be "linearized", neglecting the term $y_{n}^{n}(\xi)$ in the surface stellar region. The solution of equation $\Delta_{\xi} y_{n}(\xi)=0$ is the function

$$
y_{n}(\xi)=\beta_{2}(n)\left(\frac{1}{\xi}-\frac{1}{\xi_{1}(n)}\right) \simeq \frac{\beta_{2}(n)}{\xi_{1}^{2}(n)}\left(\xi_{1}(n)-\xi\right),
$$

which determines the asymptotic of the solution in the vicinity $\xi_{1}(n)$. Hence,

$$
\beta_{2}(n)=\int_{0}^{\xi_{1}(n)} \xi^{2} y_{n}^{n}(\xi) d \xi=\xi_{1}^{2}(n)\left|\frac{d y_{n}}{d \xi}\right|_{\xi_{1}(n)}
$$

is a characteristic parameter of the polytropic model, that determines its mass and energy. The nature of the solutions of equations (27), (28) are illustrated in Fig. 1.

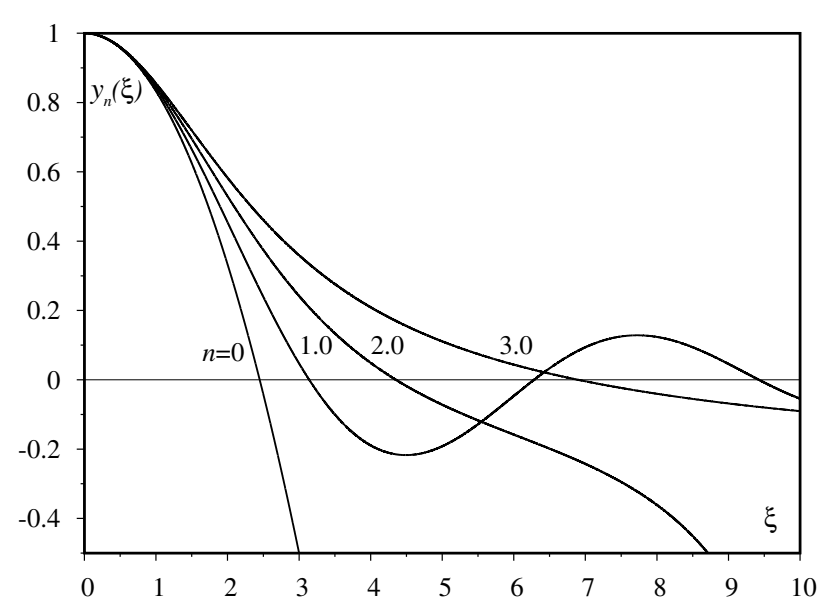

Fig. 1. The Emden solutions of equation for the polytropic index $0 \leq n \leq 3.0$

The dependencies of the values $\xi_{1}(n), \beta_{2}(n)$ and

$$
\beta_{4}(n)=\int_{0}^{\xi_{1}(n)} \xi^{4} y_{n}^{n}(\xi) d \xi
$$

on the polytropic index are shown in Table 1. 


\begin{tabular}{|c|c|c|c|c|c|c|c|}
\hline$n$ & 0 & 0.5 & 1.0 & 1.5 & 2.0 & 2.5 & 3.0 \\
\hline \hline$\xi_{1}(n)$ & 2.4495 & 2.7527 & 3.1416 & 3.6538 & 4.3529 & 5.3553 & 6.8969 \\
\hline$\beta_{2}(n)$ & 4.8990 & 3.7887 & 3.1416 & 2.7141 & 2.4111 & 2.1872 & 2.0182 \\
\hline$\beta_{4}(n)$ & 17.6371 & 14.0352 & 12.1567 & 11.1197 & 10.6110 & 10.5197 & 10.8516 \\
\hline
\end{tabular}

Table 1. The Emden's parameters of the polytropic models

As can be seen from formulae (29), the function $y_{1}(\xi)$ coincides with the spherical Bessel function of the first kind $j_{0}(\xi)$ [19]. The functions $y_{n}(\xi)$ at $n>1$ in the asymptotic $\xi \ll 1$ also coincide with the function $j_{0}(\xi)$. The solutions of equation (27) were shown in Fig. 1, these solutions are changeable functions $\xi$ : if $n$ is even, then the equation $y_{n}(\xi)=0$ has a single root $\xi_{1}(n)=\xi_{E}(n)$, in the case of an odd $n$, this equation has many roots $\xi_{m}(n)$ and the functions $y_{n}(\xi)$ are oscillating.

According to formulae (15), the mass, volume and the moment of inertia of the polytropic star without rotation are determined by the expressions:

$$
\begin{aligned}
& M(n, 0)=4 \pi \lambda_{n}^{3} \rho_{c} \beta_{2}(n) ; V(n, 0)=\frac{4 \pi}{3}\left(\lambda_{n} \xi_{1}(n)\right)^{3} ; \\
& I(n, 0)=\frac{8}{3} \pi \lambda_{n}^{5} \rho_{c} \beta_{4}(n) .
\end{aligned}
$$

\section{THE MODELS WITH AXIAL ROTATION. LINEAR EQUATIONS}

To reveal the basic differences between the solutions of equations (9), (26) and the Emden functions, we consider partial cases $n=0$ and $n=1$, when these equations are linear and allow the solutions in the analytical form. Furthermore, these partial cases illustrate the features of our technique.

\section{A. Polytrope $n=0$}

This case is almost trivial. The simple model with constant density $\left(\rho(\mathbf{r})=\rho_{c}\right)$ corresponds to an incompressible fluid and the solution of the equilibrium equation only determines the shape and size of the object. We consider dimensionless angular velocity (but not eccentricity) to be an independent parameter and we determine the mass and shape of the model surface in the region of valid values of angular velocity.

According to formulae (23), (26) at $n=0$, the solution of the equilibrium equation takes the form

$$
Y_{0}(\xi, \theta)=y_{0}(\xi)+\frac{\xi^{2}}{6} \Omega^{2}\left(1-P_{2}(t)\right)-\frac{\xi^{2}}{2} P_{2}(t) I(e)
$$

where $y_{0}(\xi)=1-\xi^{2} / 6$ is the Emden function with the polytropic index $n=0$. From the condition $Y_{0}(\xi, \theta)=0$ we find the equation for the surface

$$
\xi_{0}(\theta)=\sqrt{6}\left\{1-\Omega^{2}\left(1-P_{2}(t)\right)+3 P_{2}(t) I(e)\right\}^{-1 / 2} .
$$

Having determined the polar and equatorial radii from that formula and using the expression $1-e^{2}=\xi_{p}^{2} / \xi_{e}^{2}$ we obtain the equation

$$
e^{2}+\left(3 e^{2}-\frac{9}{2}\right) I(e)=\frac{3}{2} \Omega^{2},
$$

which is known as the Maclaurin formula [6], that determines the dependence of eccentricity on angular velocity. In the approximation $e^{2} \ll 1$ we find that

$e^{2}=\frac{15}{4} \Omega^{2}-\frac{225}{112} \Omega^{4}+\ldots, I(e(\Omega))=\frac{1}{2} \Omega^{2}-\frac{2}{15} \Omega^{4}+\ldots$,

in connection with

$$
\begin{aligned}
& \xi_{p} \simeq \sqrt{6}\left\{1-\frac{3}{4} \Omega^{2}+\frac{167}{160} \Omega^{4}+\ldots\right\}, \\
& \xi_{e} \simeq \sqrt{6}\left\{1+\frac{9}{8} \Omega^{2}+\frac{1151}{640} \Omega^{4}+\ldots\right\} .
\end{aligned}
$$

It follows also the mass and volume of the polytropic star with index $n=0$ are determined by the expression

$$
\begin{aligned}
& M(0, \Omega)=M(0,0) f(\Omega), V(0, \Omega)=V(0,0) f(\Omega) \\
& f(\Omega) \cong 1+\frac{3}{2} \Omega^{2}+\frac{11}{2} \Omega^{4}+\ldots
\end{aligned}
$$

where $M(0,0)$ and $V(0,0)$ are the mass and volume without rotation. The character of the solution of equation (37) is shown in Fig. 2 relative to the eccentricity.

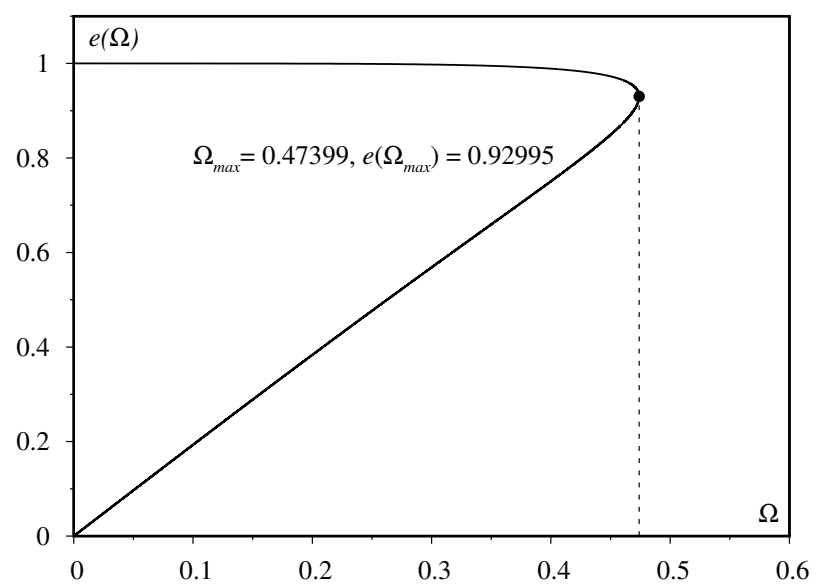

Fig. 2. Dependence of the eccentricity $e(\Omega)$ on the angular velocity $\Omega$

As was shown in Figure, the eccentricity is an ambiguous function of the parameter $\Omega$, and real solutions only exist in the region $0 \leq \Omega \leq \Omega_{\max }=0.47399 \ldots$ The value $\Omega_{\max }$ corresponds to the $e\left(\Omega_{\max }\right)=0.92995 \ldots$. The critical values $\Omega_{\max }$ and $e\left(\Omega_{\max }\right)$ determine the 
instability point with the polytropic index $n=0$ relative to the rotation velocity $[6,20]$. The configurations which belong to the region $0<e \leq e\left(\Omega_{\max }\right)$ are typical ellipsoidal structures, and those which belong to the range $e\left(\Omega_{\max }\right)<e \leq 1.0$ are similar to disk structures. The dependence $I(\Omega) \equiv I(e(\Omega))$ on rotational velocity is shown in Fig. 3. Here, $I\left(e\left(\Omega_{\max }\right)\right)=0.27698$. The solid

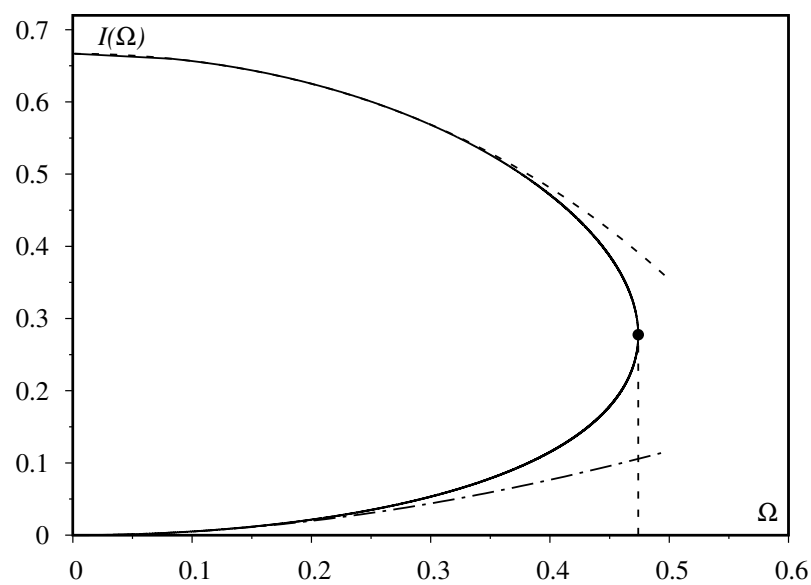

Fig. 3. Dependence of the function $I(\Omega)$ on angular velocity $\Omega$. Dashed-dot curve corresponds to small velocity (small value of the eccentricity), dashed - small velocity at large values of the eccentricity

curve in Fig. 3 corresponds to the dependence, which was depicted in Fig. 2.

The dimensionless equatorial and polar radii are determined from expression (36)

$$
\begin{aligned}
& \xi_{e}(\Omega)=\sqrt{6}\left\{1-\frac{3}{2}\left[\Omega^{2}+I(\Omega)\right]\right\}^{-1 / 2}, \\
& \xi_{p}(\Omega)=\sqrt{6}\{1+3 I(\Omega)\}^{-1 / 2}
\end{aligned}
$$

are shown in Fig. 4. The solid curves correspond to the

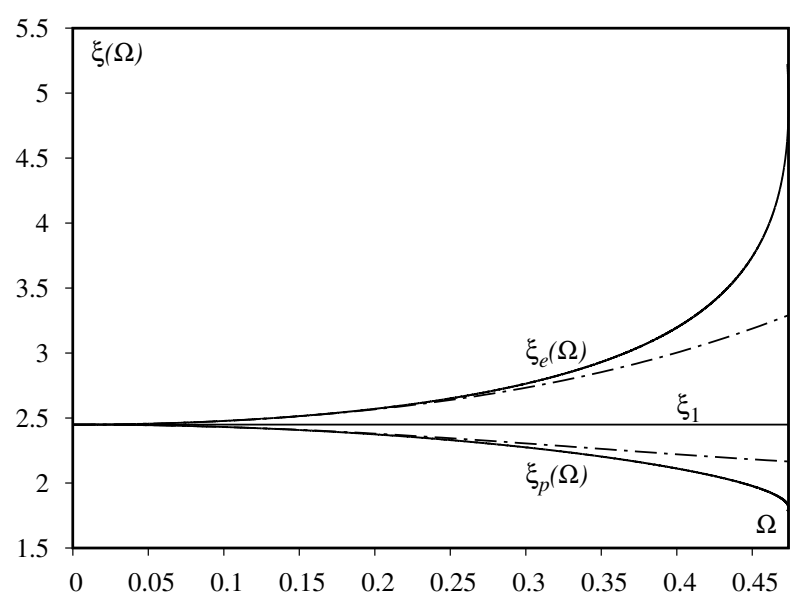

Fig. 4. Dependencies of equatorial $\xi_{e}(\Omega)$ and polar $\xi_{p}(\Omega)$ radii on angular velocity $\Omega$ (the dashed-dot curve corresponds to small velocity)

numerical calculations in the region $0 \leq e \leq e\left(\Omega_{\max }\right)$ and the dashed-dot curves - the approximation of small velocities. As was shown in the figure, the approximation of small velocities is applicable in the range $\Omega \leq 0.5 \Omega_{\max }$.

Dependence of the stellar mass on rotational velocity

$$
M(0, \Omega)=M(0,0) \xi_{e}^{2}(\Omega) \xi_{p}(\Omega) \xi_{1}^{-3}(0)
$$

is shown in Fig. 5. Here the dashed-dot curve corresponds to small velocity according to the formula (40).

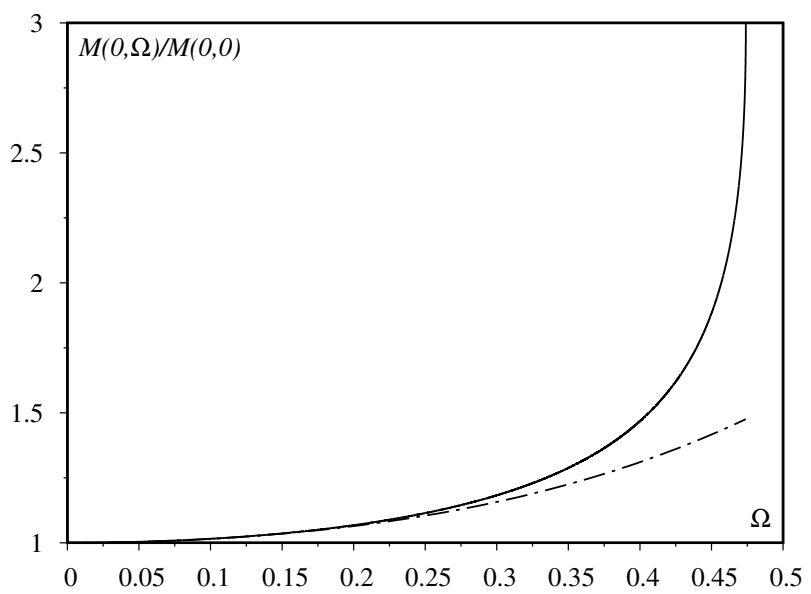

Fig. 5. Dependence of the stellar polytropic mass on the rotational velocity $\Omega$ (the dashed-dot curve corresponds to formula (40))

In the range $e\left(\Omega_{\max }\right) \leq e \leq 1$

$$
I(\Omega) \approx \frac{2}{3}-\Omega^{2}-\Omega^{4}+\ldots,
$$

which is shown with the dashed curve in Fig. 3. Therefore, according to formulae (41) in the region of large values of the eccentricity and small values of rotational velocity

$$
\xi_{e}(\Omega) \Rightarrow \frac{2}{\Omega^{2}}+\ldots, \quad \xi_{p}(\Omega) \Rightarrow \sqrt{2}
$$

which corresponds to the disk of constant thickness and a large radius.

In the work [12] the solution of the equation (9) at $n=0$ is written in the form

$$
Y(\xi, \theta)=1-\frac{\xi^{2}}{6}\left(1-\Omega^{2}\right)+B_{2} \xi^{2} P_{2}(t),
$$

and the integration constant $B_{2}$ is determined from expression (19), that connects the solution of the equilibrium equation with the gravitational potential, which at $n=0$ is calculated accurately. The dependence of the polytropic characteristics on the parameter $0 \leq$ $\Omega \leq \Omega_{\max }$ and the parameter $\varepsilon=\left(1-e^{2}\right)^{1 / 2}=\xi_{p} / \xi_{e}$ is tabulated in the region $1 \geq \varepsilon \geq 0$.

\section{B. Polytrope $n=1$.}

The polytrope with index $n=1$ plays the role of a certain standard: although the exact analytical solution of equation (9) is unknown, the latter is linear and this 
allows us to find the approximate solutions with high precision. In this case, it is convenient to rewrite equation (9) in the form of the following inhomogeneous equation

$$
\begin{aligned}
& \Delta(\xi, \theta) \varphi(\xi, \theta)+\varphi(\xi, \theta)=-\frac{\Omega^{2}}{4} \xi^{2} \sin ^{2} \theta \\
& \varphi(\xi, \theta)=Y_{1}(\xi, \theta)-\frac{\Omega^{2}}{4} \xi^{2} \sin ^{2} \theta
\end{aligned}
$$

In the corresponding homogeneous equation, the variables are separated and its general solution

$$
\varphi(\xi, \theta)=\sum_{l=0}^{\infty} B_{2 l} j_{2 l}(\xi) P_{2 l}(t)
$$

where $j_{2 l}(\xi)$ is the spherical Bessel function of the first kind [19], $B_{2 l}$ are the integration constants. The substitution $\varphi(\xi, \theta)=\Omega^{2} \tilde{\varphi}(\xi, \theta)$ allows us to get rid of the parameter $\Omega^{2}$ in equation (46):

$$
\Delta(\xi, \theta) \tilde{\varphi}(\xi, \theta)+\tilde{\varphi}(\xi, \theta)=-\frac{\xi^{2}}{4} \sin ^{2} \theta .
$$

We find a partial solution of this equation in the form

$$
\tilde{\varphi}(\xi, \theta)=\sum_{l=2}^{\infty} b_{2 l}[\xi \sin \theta]^{2 l}
$$

Using the equality

$$
\Delta(\xi, \theta)\{\xi \sin \theta\}^{2 l}=(2 l)^{2}\{\xi \sin \theta\}^{2 l-2},
$$

we find that

$$
b_{2 l}=(-1)^{l-1} 2^{-2 l}(l !)^{-2}
$$

therefore

$$
\frac{1}{4} \xi^{2} \sin ^{2} \theta+\tilde{\varphi}(\xi, \theta)=1-J_{0}(\xi \sin \theta)
$$

where $J_{0}(z)$ is the Bessel function of the zero kind [19]. From the differential equation for the function $Y_{1}(\xi, \theta)$ the asymptotic behavior follows

$$
Y_{1}(\xi, \theta) \Rightarrow 1-\frac{\xi^{2}}{6}+\frac{\Omega^{2} \xi^{2}}{4} \sin ^{2} \theta+\ldots
$$

at $\xi \rightarrow 0$; as a result the general solution of equation (9), which corresponds to the boundary conditions at $\xi=0$, can be represented in the form

$$
Y_{1}(\xi, \theta)=j_{0}(\xi)+\Omega^{2}\left\{1-J_{0}\left(\xi\left[1-t^{2}\right]^{1 / 2}\right)+\sum_{l=1}^{\infty} \alpha_{2 l} j_{2 l}(\xi) P_{2 l}(t)\right\}
$$

where $\alpha_{2 l}$ are the integration constants. In expression (54) together with the fundamental type solutions $j_{2 l}(\xi) \cdot P_{2 l}(t)$, that arise from the separation of the variables (and which were mentioned by Z. Kopal [9]), the term $\Omega^{2}\left(1-J_{0}(\xi[1-\right.$ $\left.\left.t^{2}\right]^{1 / 2}\right)$ appears. The function $J_{0}\left(\xi\left[1-t^{2}\right]^{1 / 2}\right)$ has the following expansion for the Legendre polynomials $(t=\cos \theta)$ and the spherical Bessel functions [19]:

$$
J_{0}\left(\xi\left[1-t^{2}\right]^{1 / 2}\right)=\sum_{l=0}^{\infty} D_{l} j_{2 l}(\xi) P_{2 l}(t), D_{l}=(4 l+1)(2 l) ! 2^{-2 l}(l !)^{-2} .
$$

Thereby the solution can be represented in the form of a series for the orthogonal functions

$$
Y_{1}(\xi, \theta)=j_{0}(\xi)+\Omega^{2}\left(1-j_{0}(\xi)\right)+\Omega^{2} \sum_{l=1}^{\infty} a_{2 l} j_{2 l}(\xi) P_{2 l}(t) .
$$

Such representation was proposed in the work [16]. In the practical calculations, we restricted ourselves to the terms $1 \leq l \leq 3$, and the integration constants $a_{2 l}$ were determined from equation (26).

Formally, taking into account the infinite number of the series terms for the Legendre polynomials, representations (54) and (56) are completely equivalent. However, in practical calculations only a small number of terms should be taken into account. In this case, representations (54) and (56) are not already equivalent. This is due to the features of angular dependence of the function $J_{0}\left(\xi\left[1-t^{2}\right]^{1 / 2}\right)$ :

$$
\begin{aligned}
& \lim _{t \rightarrow \pm 1} J_{0}\left(\xi\left[1-t^{2}\right]^{1 / 2}\right)=1 ; \\
& \lim _{t \rightarrow 0} J_{0}\left(\xi\left[1-t^{2}\right]^{1 / 2}\right)=J_{0}(\xi) ; \quad \frac{1}{2} \int_{-1}^{+1} J_{0}\left(\xi\left[1-t^{2}\right]^{1 / 2}\right) d t=j_{0}(\xi) .
\end{aligned}
$$


Thereby the angular dependence of the function

$$
Y_{1}(\xi, \theta)=j_{0}(\xi)+\Omega^{2}\left\{1-J_{0}\left(\xi\left[1-t^{2}\right]^{1 / 2}\right)+\sum_{l=1}^{l_{0}} \alpha_{2 l} j_{2 l}(\xi) P_{2 l}(t)\right\},
$$

and the function

$$
\tilde{Y}_{1}(\xi, \theta)=j_{0}(\xi)+\Omega^{2}\left\{1-j_{0}(\xi)+\sum_{l=1}^{l_{0}} a_{2 l} j_{2 l}(\xi) P_{2 l}(t)\right\}
$$

are different. The term $1-J_{0}\left(\xi\left[1-t^{2}\right]^{1 / 2}\right)$ is the result of the selective summation of the infinite series in formula (56). This term reflects the natural asymmetry of the solution in the polar and the equatorial directions. First of all, we consider the case of the calculation based on function (58), which is different from all the representations of the solution of the equilibrium equation for the polytrope with index $n=1$, which are used by other authors.

The integration constants $\alpha_{2 l}$ will be found from expression (26) for the case $n=1$,

$$
\begin{aligned}
Y_{1}(\xi, \theta) & =1+\frac{\Omega^{2} \xi^{2}}{6}\left(1-P_{2}(t)\right) \\
& +\frac{1}{4 \pi} \int Y_{1}\left(\xi^{\prime}, \theta^{\prime}\right) Q\left(\boldsymbol{\xi}, \boldsymbol{\xi}^{\prime}\right) d \boldsymbol{\xi}^{\prime} .
\end{aligned}
$$

Substituting expression (58) into it and taking into account (28), as well as the fact, that $J_{0}\left(\xi\left[1-t^{2}\right]^{1 / 2}\right)$ is the partial solution of equation (46), we obtain the relation

$$
\begin{aligned}
& \sum_{l=1}^{l_{0}} \alpha_{2 l} j_{2 l}(\xi) P_{2 l}(t)=-P_{2}(t) \frac{\xi^{2}}{6}\{1+3 I(e)\} \\
& +\frac{1}{4 \pi} \sum_{l=1}^{l_{0}} \alpha_{2 l} \int j_{2 l}\left(\xi^{\prime}\right) P_{2 l}\left(t^{\prime}\right) Q\left(\boldsymbol{\xi}, \boldsymbol{\xi}^{\prime}\right) d \boldsymbol{\xi}^{\prime} .
\end{aligned}
$$

We will perform integration over variables $\xi^{\prime}, t^{\prime}, \varphi^{\prime}$ in the form of the rotational ellipsoid model with the eccentricity $e$ and the equatorial radius $\xi_{e}$, as in the case of $n=0$, expanding the kernel $Q\left(\boldsymbol{\xi}, \boldsymbol{\xi}^{\prime}\right)$ in a series of the Legendre polynomials:

$$
\begin{aligned}
& \frac{1}{4 \pi} \sum_{l=1}^{l_{0}} \alpha_{2 l} \int j_{2 l}\left(\xi^{\prime}\right) P_{2 l}\left(t^{\prime}\right) Q\left(\boldsymbol{\xi}, \boldsymbol{\xi}^{\prime}\right) d \boldsymbol{\xi}^{\prime}=\sum_{l=1}^{l_{0}} \frac{\alpha_{2 l}}{4 l+1} \frac{P_{2 l}(t)}{\xi^{1+2 l}} \int_{0}^{\xi}\left(\xi^{\prime}\right)^{2+2 l} j_{2 l}\left(\xi^{\prime}\right) d \xi^{\prime} \\
& +\frac{1}{2} \sum_{l=1}^{l_{0}} \alpha_{2 l} P_{2 l}(t) \xi^{2 l} \int_{-1}^{+1} P_{2 l}^{2}\left(t^{\prime}\right) d t^{\prime} \int_{\xi}^{\xi_{0}\left(t^{\prime}\right)} j_{2 l}\left(\xi^{\prime}\right)\left(\xi^{\prime}\right)^{1-2 l} d \xi^{\prime} \\
& +\frac{1}{2} \sum_{l, m=1}^{l_{0}} \alpha_{2 l} P_{2 m}(t) \xi^{2 m}\left(1-\delta_{m, l}\right) \int_{-1}^{+1} P_{2 l}\left(t^{\prime}\right) P_{2 m}\left(t^{\prime}\right) d t^{\prime} \int_{\xi}^{\xi_{0}\left(t^{\prime}\right)} j_{2 l}\left(\xi^{\prime}\right)\left(\xi^{\prime}\right)^{1-2 m} d \xi^{\prime},
\end{aligned}
$$

where $\delta_{n, l}$ is the Kronecker symbol and $\xi_{0}\left(t^{\prime}\right)$ is determined by formula (22).

Integration over the variable $\xi^{\prime}$ is performed in the analytical form using the equation for the function $j_{2 l}(\xi)$, as well as the following recurrent formulae [19]

$$
\begin{aligned}
& j_{2 l}=-\xi^{2 l-1} \frac{d}{d \xi}\left\{\xi^{1-2 l} j_{2 l-1}(\xi)\right\} ; \\
& (4 l+1) j_{2 l}(\xi)=\xi\left\{j_{2 l+1}(\xi)+j_{2 l-1}(\xi)\right\} \\
& \frac{d}{d \xi} j_{l}(\xi)=j_{l-1}(\xi)-\frac{l+1}{\xi} j_{l}(\xi) ; \quad l \geq 1 .
\end{aligned}
$$

Comparing the coefficients at the same factors $\xi^{2 l} P_{2 l}(t)$ in the left and right sides of expression (61), we obtain the following system of linear equations for the constants $\alpha_{2 l}$ :

$$
\begin{aligned}
& \alpha_{2} S_{2,2}+\alpha_{4} S_{2,4}+\ldots+\alpha_{2 l_{0}} S_{2,2 l_{0}}=-\frac{1}{6}(1+3 I(e)) ; \\
& \alpha_{2} S_{4,2}+\alpha_{4} S_{4,4}+\ldots+\alpha_{2 l_{0}} S_{4,2 l_{0}}=0 \\
& \ldots \ldots \ldots \ldots \ldots \ldots \ldots \ldots \ldots \ldots \ldots \ldots \\
& \alpha_{2} S_{2 l_{0}, 2}+\alpha_{4} S_{2 l_{0}, 4}+\ldots+\alpha_{2 l_{0}} S_{2 l_{0}, 2 l_{0}}=0 .
\end{aligned}
$$




\begin{tabular}{|c|c|c|c|c|c|c|c|}
\hline$\Omega$ & $e(\Omega)$ & $\xi_{p}(\Omega)$ & $\xi_{e}(\Omega)$ & $\alpha_{2}(\Omega)$ & $\alpha_{4}(\Omega)$ & $\eta(n, \Omega)$ & $\zeta(n, \Omega)$ \\
\hline 01000 & 0.03181 & 3.14081 & 3.14240 & -8.22777 & 0.00823046 & 1.00023 & 1.00069 \\
\hline 2000 & 06357 & 3.13845 & 3.14481 & -8.23709 & 826 & 1.00092 & 1.00276 \\
\hline 03000 & 9529 & 3.13453 & 1488 & -8.25271 & 0.0744407 & 1.00207 & 1.00624 \\
\hline 04000 & 12692 & 3.12906 & 3.15457 & -8.2 & 18 & 1.0037 & 1.01115 \\
\hline 05000 & 5846 & 3.12203 & 3.16198 & -8.3 & & 582 & 1.01755 \\
\hline .06000 & 18989 & 3.11347 & 3.17117 & -8.33901 & 0.302896 & 1.00845 & 1.02550 \\
\hline 7000 & 2120 & 3.10338 & 3.182 & -8.3 & 83 & 1.0 & 1.03509 \\
\hline 0.08000 & 25234 & 3.09179 & 3.19519 & -8.43219 & 0.548513 & 1.01531 & 1.04642 \\
\hline 09000 & 28334 & 3.07869 & 3.21025 & -8.4 & 305 & 962 & 1.05962 \\
\hline 0.10000 & 31417 & 3.06410 & 3.22752 & -8.5 & 367 & 1.02456 & 1.07486 \\
\hline 11000 & 34483 & 3.04803 & 3.24720 & -8.6 & 946 & 018 & 1.09232 \\
\hline 12000 & 37532 & 3.03048 & 3.26950 & -8.7 & & 654 & 224 \\
\hline 13000 & 40567 & 3.01144 & 3.29472 & -8.8 & 409 & 1.04373 & 1.13493 \\
\hline 1000 & 13586 & 091 & 3.323 & -8.9 & & & 1.1 \\
\hline 0.15000 & 0.46596 & 2.96885 & 3.35536 & -9.06006 & 2.18236 & 1.06092 & 1.19013 \\
\hline 0.16000 & 49598 & 2.94521 & 3.39180 & -9.2 & & 117 & 1.22371 \\
\hline 0.17000 & 0.52601 & 2.91993 & 3.43328 & -9.36930 & 2.97691 & 1.08275 & 1.26221 \\
\hline 18000 & 613 & 2.89289 & 3.48081 & -9.5 & & & 1.3066 \\
\hline 0.19000 & 0.58648 & 2.86392 & 3.53586 & -9.77991 & 4.02346 & 1.11085 & 1.35845 \\
\hline 0000 & 61728 & 2.83276 & 3.60061 & -10.04100 & 4.68 & 1.12807 & 1.41956 \\
\hline 0.21000 & 64889 & 2.79896 & 3.67856 & -10.35720 & 5.47905 & 1.14816 & 1.49304 \\
\hline 0.22000 & .68197 & 2.76174 & 3.77604 & -10.75550 & 6.47174 & 1.17208 & 1.58394 \\
\hline 0.23000 & 71801 & 2.71936 & 3.90695 & -11.29530 & 7.80005 & 1.20165 & 1.70216 \\
\hline 0.24000 & 0.76241 & 2.66599 & 4.11998 & -12.18550 & 9.9398 & 1.24181 & 1.87604 \\
\hline 0.24100 & 76815 & 2.65918 & 4.15324 & -12.32580 & 10.2704 & 1.24703 & 1.90011 \\
\hline 0.24200 & 0.77450 & 2.65173 & 4.19196 & -12.48960 & 10.654 & 1.25269 & 1.92679 \\
\hline 0.24300 & 78185 & 2.64324 & 4.23955 & -12.69170 & 11.1 & 1.25902 & 1.95741 \\
\hline 0.24400 & 0.79133 & 2.63259 & 4.30595 & -12.97500 & 11.7734 & 1.26661 & 1.99561 \\
\hline 0.24410 & .79253 & 2.63127 & 4.31477 & -13.01280 & 11.8594 & 1.26751 & 2.00026 \\
\hline 0.24420 & 0.79382 & 2.62987 & 4.32435 & -13.05380 & 11.9526 & 1.26845 & 2.00517 \\
\hline 0.24430 & 0.79522 & 2.62836 & 4.33488 & -13.09900 & 12.055 & 1.26945 & 2.01043 \\
\hline 0.24440 & 0.79676 & 2.62671 & 4.34673 & -13.14990 & 12.170 & 1.27052 & 2.01616 \\
\hline 0.24450 & 0.79853 & 2.62484 & 4.36049 & -13.20900 & 12.3033 & 1.2717 & 2.02254 \\
\hline 0.24460 & 0.80067 & 2.62259 & 4.37754 & -13.28240 & 12.4681 & 1.27307 & 2.03005 \\
\hline 0.24470 & 0.80375 & 2.61944 & 4.40267 & -13.39080 & 12.7103 & 1.27488 & 2.04028 \\
\hline
\end{tabular}

Table 2. Dependence of the model characteristics with index $n=1$ on angular velocity in the approximation (58)

The coefficients $S_{2 l, 2 l}, S_{2 m, 2 l}, \ldots$ are functions of the parameters $e, \xi_{e}$ and are determined by such expressions:

$$
\begin{aligned}
S_{2 l, 2 l} & =\int_{0}^{1} P_{2 l}^{2}(t) \xi_{0}^{1-2 l} j_{2 l-1}\left(\xi_{0}\right) d t \\
S_{2 m, 2 l} & =-\int_{0}^{1} P_{2 m}(t) P_{2 l}(t)\left\{\int_{\xi_{1}(n)}^{\xi_{0}}\left(\xi^{\prime}\right)^{1-2 m} j_{2 l}\left(\xi^{\prime}\right) d \xi^{\prime}\right\} d t .
\end{aligned}
$$

The expressions $S_{2 m, 2 l}$ are reduced to single integrals:

$$
\begin{aligned}
S_{2,4}= & \int_{0}^{1} P_{2}(t) P_{4}(t) \xi_{0}^{-1}\left\{j_{3}\left(\xi_{0}\right)+2 \xi_{0}^{-1} j_{2}\left(\xi_{0}\right)\right\} d t \\
S_{2,6}= & \int_{0}^{1} P_{2}(t) P_{6}(t) \xi_{0}^{-1}\left\{j_{5}\left(\xi_{0}\right)+4 \xi_{0}^{-1} j_{4}\left(\xi_{0}\right)+8 \xi_{0}^{-2} j_{3}\left(\xi_{0}\right)\right\} d t ; \\
S_{4,6}= & \int_{0}^{1} P_{4}(t) P_{6}(t) \xi_{0}^{-3}\left\{j_{5}\left(\xi_{0}\right)+2 \xi_{0}^{-1} j_{4}\left(\xi_{0}\right)\right\} d t \\
& \ldots \ldots \ldots \ldots \ldots \ldots \ldots \ldots \ldots \ldots \ldots \ldots \ldots \ldots \ldots
\end{aligned}
$$


Here $\xi_{0} \equiv \xi_{0}(t)$ is determined by formula (22). As was shown by expressions (65), at small values of angular velocity

$$
\begin{aligned}
& S_{2 l, 2 l} \approx \tilde{S}_{2 l, 2 l}=(4 l+1)^{-1} \xi_{1}^{1-2 l} j_{2 l-1}\left(\xi_{1}\right), \\
& \alpha_{2} \approx-\frac{5}{6} \xi_{1}^{2}, \alpha_{2 l}=0 \text { at } l \geq 2,
\end{aligned}
$$

where $\xi_{1}=\pi$ is the dimensionless radius in the Emden approximation. The constant $\alpha_{2}$ does not depend on the rotation velocity and corresponds to the approximation of the works $[8,11]$. This is a raw approximation, which is suitable for the small angular velocities.

The coefficients $\alpha_{2 l}$ are the functions of angular velocity, as well as the eccentricity $e$ and the equatorial radius $\xi_{e}$, which are the same functions of angular velocity. Therefore, there arises a problem of the self-consistent determining of geometrical parameters of the rotational ellipsoid. We performed the practical calculations in the approximation $\alpha_{2 l}=0$ at $l \geq 3$. The condition

$$
\begin{aligned}
j_{0}\left(\xi_{0}\right) & +\Omega^{2}\left\{1-J_{0}\left(\xi_{0}\left[1-t^{2}\right]^{1 / 2}\right)\right. \\
& \left.+\sum_{l=1}^{2} \alpha_{2 l} j_{2 l}\left(\xi_{0}\right) P_{2 l}(t)\right\}=0
\end{aligned}
$$

determines the equation of the stellar surface $\xi_{0}(t) \equiv$ $\xi_{0}(t \mid \Omega)$. The root of equation at $t=1$ determines the polar radius $\xi_{p}(\Omega) \equiv \xi_{0}(1 \mid \Omega)$ and the root at $t=0$ yields the equatorial radius $\xi_{e}(\Omega) \equiv \xi_{0}(0 \mid \Omega)$ at $0 \leq \Omega \leq \Omega_{\max }$. The equation

$$
e^{2}(\Omega)=1-\left[\frac{\xi_{0}(1 \mid \Omega)}{\xi_{0}(0 \mid \Omega)}\right]^{2}
$$

determines the dependence of eccentricity $e(\Omega)$ on angular velocity. The system of equations (64) - (69), in which $\Omega$ is an independent parameter, determines the dependencies $e(\Omega), \xi_{e}(\Omega), \xi_{p}(\Omega)$ and $\alpha_{2 l}(\Omega)(1 \leq l \leq 2)$ on angular velocity. The system can be solved numerically by the method of successive approximations. The algorithm of successive iterations are as follows. At the initial value $\Omega_{1} \ll 1$ in zero approximation, values of $\xi_{e}(\Omega)=\xi_{p}(\Omega)$ are determined from equation (68) at $\alpha_{2}=\tilde{\alpha}_{2}, \alpha_{4}=0$. Next we find the values $S_{2 l, 2 l}, S_{2 m, 2 l}$ and solve the system of equations (64). In the next iteration we find $\xi_{p}(\Omega)$ and $\xi_{e}(\Omega)$ from equation (68) with help of coefficients $\alpha_{2 l}$ found in previous step and calculate the eccentricity $e(\Omega)$. We calculate again $S_{2 l, 2 l}, S_{2 m, 2 l}$ and etc. The final values $\alpha_{2 l}\left(\Omega_{1}\right)$ are used as the zero successive approximation for the calculation of the characteristics at $\Omega_{2}=\Omega_{1}+\Delta \Omega$ and etc. Obtained in this way, the integration constants and the ellipsoid's characteristics are shown in Table 2.

The maximal value of the angular velocity is determined from condition (11). This is an instability point, at which a leakage of the matter from the equatorial region occurs.

The dependence of the values $e(\Omega), \xi_{e}(\Omega), \xi_{p}(\Omega)$,
$\alpha_{2}(\Omega), \alpha_{4}(\Omega)$, and also the terms

$$
\begin{aligned}
& \eta(1, \Omega)=M(1, \Omega) / M(1,0), \\
& \zeta(1, \Omega)=I(1, \Omega) / I(1,0)
\end{aligned}
$$

on the the angular velocity are given in Table 2 . Here $M(1, \Omega)$ and $I(1, \Omega)$ denote the mass and the moment of inertia of the rotational ellipsoid in the accepted approximation, $M(1,0)$ and $I(1,0)$ are the mass and the moment of inertia of the polytropic star without rotation.

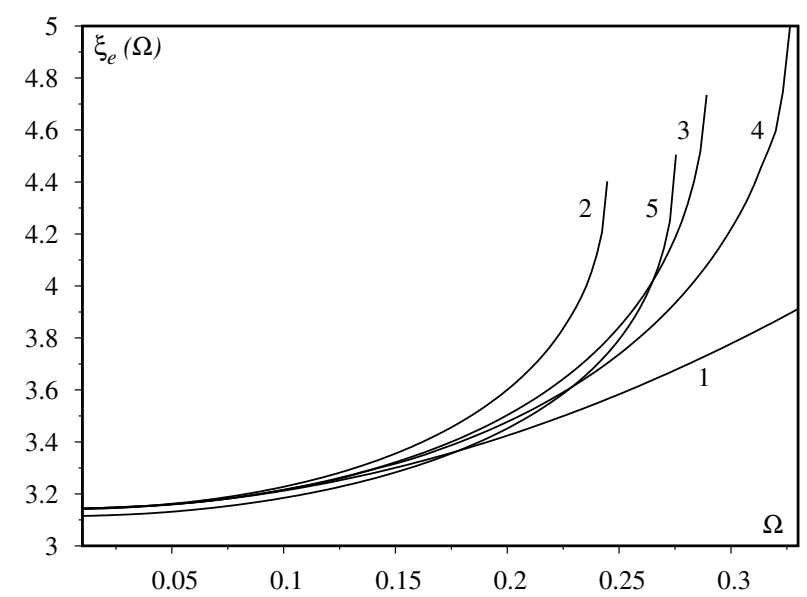

Fig. 6. Dependence of the equatorial radius $\xi_{e}(\Omega)$ on the rotation velocity $\Omega$ for the polytrope with $n=1$ in different approximations. Curve 1 is based on the results of the work [8], curve 2 corresponds to approximation (58) at $l_{0}=2$. Curve 3 is based on the results of the work [10], curve 4 - of the work [12], curve 5 - of the work [11]

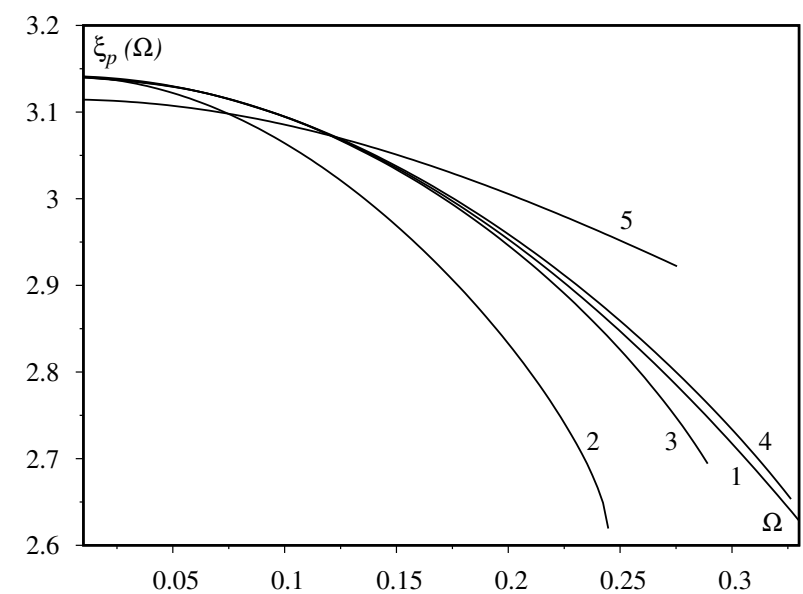

Fig. 7. Dependence of the polar radius $\xi_{p}(\Omega)$ on the rotation velocity $\Omega$ for the polytrope with $n=1$ in different approximations. Curve 1 is based on the results of the work [8], curve 2 corresponds to the approximation (58) at $l_{0}=2$. Curve 3 is based on the results of the work [10], curve 4 - of the work [12], curve 5 - of the work [11]

The dependence of the equatorial radius on the axial velocity in different approximations is illustrated in Fig. 6. Similarly, the dependence of the polar radius on 
the axial velocity in the same approximations is given in Fig. 7 .

As can be seen from the Figures, the E. Milne $\mathrm{S}$. Chandrasekhar approximation is usable in the vicinity $0 \leq \Omega \lesssim 0.5 \Omega_{\max }(1)$.

As was shown in Table 2 , the polytrope with index $n=1$ is stable in the region $0 \leq \Omega \leq \Omega_{\max }(1) \approx$ $0.2447 \ldots$ The constants $\alpha_{2}$ and $\alpha_{4}$ have opposite signs and significantly depend on angular velocity. In the region $0 \leq \Omega \leq 0.5 \Omega_{\max }(1)$ the constant $\alpha_{4}$ is small, and in the region $\Omega>0.5 \Omega_{\max }(1)$ it is close to the $\left|\alpha_{2}\right|$. Since in the region $0 \leq \xi \leq \xi_{e}\left(\Omega_{\max }\right)$ the functions $j_{2}(\xi)$ and $j_{4}(\xi)$ are positive, the approximation $\alpha_{4}=0$ is only applicable in the region of small velocities $\Omega$.

Approximation (59) at $l_{0}=1$ corresponds to works [8] and [12]. In the work [8] the constant $\alpha_{2}$ is determined by expression (67) at $\xi_{1}=\pi$, and in the work [12] it is calculated numerically based on the calculati- on of the change of the gravitational potential, which is caused by the rotation. The critical parameters of angular velocity, which are given by the authors are: $\Omega_{\max }(1) \approx 0.3315$ [8] and $\Omega_{\max }(1) \approx 0.3264 \ldots$ [12]. The author [12] mistakenly considered approximation (59) at $l=1$ as the accurate solution of the problem. In fact, the results obtained by him improve the results of the work [8]. The approximation of S. Chandrasekhar [8] is used also in the work [11] for the description of the inner polytrope region, whose radius is close to the Emden radius. It was found that the maximal value of the velocity $\Omega_{\max }(1) \approx 0.2755$. In the work [10], the polytrope characteristics are calculated numerically. The index $n=1$ corresponds to the $\Omega_{\max }(1) \cong 0.2891 \ldots$. From these comparisons, it is possible to conclude: the more precise the calculation, the smaller the value of the critical velocity $\Omega_{\max }$.

\begin{tabular}{|c|c|c|c|c|c|c|c|c|}
\hline$\Omega$ & $e(\Omega)$ & $\xi_{p}(\Omega)$ & $\xi_{e}(\Omega)$ & $a_{2}(\Omega)$ & $a_{4}(\Omega)$ & $a_{6}(\Omega)$ & $\eta(n, \Omega)$ & $\zeta(n, \Omega)$ \\
\hline 0.01000 & .02739 & 3.14112 & 3.14230 & -8.22784 & 0.00610775 & $-8.02713 \cdot 10^{-6}$ & 1.00023 & 1.00062 \\
\hline 2000 & 5478 & 3971 & 14443 & & & & 1.00092 & 1.00 \\
\hline 3000 & 8219 & 3734 & 3.14799 & 8.25338 & & 43 & 1.00207 & 1.005 \\
\hline 94000 & 10961 & 13402 & 15302 & 8.27594 & 0989151 & -0 . & 1.00369 & 1.01006 \\
\hline 0.05000 & 13706 & 12973 & 3.15955 & -8.30523 & 155695 & & 1.00580 & 1.0158 \\
\hline 6000 & 16455 & 12447 & 3.16765 & 51 & & & & 1.02298 \\
\hline 07000 & 19208 & 3.11820 & 3.17737 & -8.38505 & 311294 & 555 & 1.01150 & 1.03158 \\
\hline 08000 & 21967 & 11092 & 3.18880 & -8.43625 & 0.411773 & -0 . & 1.01513 & 1.04172 \\
\hline 9000 & 24733 & 10259 & 3.20205 & -8.4 & & & & 1.05 \\
\hline 000 & 27507 & . 09318 & 3.21725 & -8.56 & & & & \\
\hline 11000 & 30291 & 3.08266 & 3.23456 & -8.6 & 398 & & 1.02951 & 1.08256 \\
\hline 2000 & 33087 & 3.07097 & 3.25416 & -8.7 & & & & 1.10016 \\
\hline 3000 & 5900 & 05807 & 3.27632 & -8.8 & & & & 1.12011 \\
\hline 0.14000 & 38731 & 04388 & 3.30131 & -8.93941 & 314 & & 1.04994 & 1.14270 \\
\hline 15000 & 41586 & 02832 & 3.32953 & -9.0 & & & 839 & 1.16830 \\
\hline 6000 & 14471 & 01127 & 3.36147 & -9.2 & & & & 1.19736 \\
\hline 17000 & 47394 & 99259 & 3.39779 & -9.3 & & & 834 & 1.23047 \\
\hline 0.18000 & 50367 & 2.97208 & 3.43938 & -9.5 & & & 1.09014 & 1.26843 \\
\hline 9000 & 53407 & 94946 & 3.48752 & -9. & & & & 1. \\
\hline 0.20000 & 56538 & 2.92430 & 3.54414 & -10.02570 & 946 & & 855 & 1.36371 \\
\hline 0.21000 & 59802 & 2.89594 & 3.61237 & -10.33050 & & & 1.13597 & 1.42496 \\
\hline 22000 & 63273 & 86321 & 3.69793 & -10.7 & & & & 1.50007 \\
\hline 0.23000 & 67114 & 82368 & 3.81334 & -11.2 & 235 & & 1.18158 & 1.59696 \\
\hline 24000 & 71852 & 77019 & 4.00008 & -12.01930 & 79 & & 544 & 1.73805 \\
\hline 0.24100 & 0.72446 & 2.76320 & 4.02826 & -12.13670 & 357 & & 1.21980 & 1.75727 \\
\hline 0.24200 & 73086 & 2.75562 & 4.06018 & -12.26860 & 224 & -9 & 1.22449 & 1.77830 \\
\hline 0.24300 & 73793 & .74724 & 4.09737 & -12.42040 & 8.71955 & & 1.22964 & 1.80179 \\
\hline 0.24400 & 74604 & 73767 & 4.14281 & -12.60320 & & & 1.23546 & 1.82894 \\
\hline 0.24500 & 75612 & .72593 & 4.20403 & 12.8 & & & 4249 & 1.86270 \\
\hline 0.24600 & 0.77450 & 2.70593 & 4.33124 & -13.32470 & .4868 & & 1.25413 & 1.92196 \\
\hline 0.24601 & 77507 & 2.70536 & 4.33555 & -13.34070 & & & .25445 & 1.92369 \\
\hline 0.24602 & 0.77563 & 2.70481 & 4.33977 & -13.35610 & .5455 & & 1.25477 & 1.92537 \\
\hline 0.24603 & 0.77626 & 2.70418 & 4.34461 & -13.37360 & & & 1.25512 & 1.92728 \\
\hline 0.24604 & 0.77702 & 2.70344 & 4.35043 & -13.39470 & 6177 & & 1.25554 & 1.92955 \\
\hline 0.24605 & 0.77800 & 2.70249 & 4.35808 & -13.42230 & 669 & & 1.25608 & 1.93248 \\
\hline 24606 & 77959 & 2.70100 & 4.37053 & -13.4 & & & 1.25693 & 1.93714 \\
\hline 0.24607 & 0.78685 & 2.69478 & 4.42985 & -13.66320 & 11.1084 & -15.5733 & 1.26065 & 1.95773 \\
\hline
\end{tabular}

Table 3. Dependence of the model characteristics with index $n=1$ on angular velocity in the approximation (59) 
We also calculated the characteristics of the polytrope with index $n=1$ based on approximation (59) at $l_{0}=3$. The integration constants $\alpha_{2 l}$ in this variant are determined in the same way as in the case of function (58). The results of the calculation of the polytrope characteristics are shown in Table 3 . The critical parameters of the polytrope were as follows: $\Omega_{\max }(1) \cong 0.24607, \xi_{p}\left(\Omega_{\max }\right) \cong 2.69478 \ldots, \xi_{e}\left(\Omega_{\max }\right) \approx$ $4.42985 \ldots$. .

Here $\alpha_{4}>0, \alpha_{6}<0$, in the region of small velocities they are small, and near the critical point, the absolute values of all constants are similar.

The characteristics of the polytrope, which are calculated based on functions (58) and (59) are very close. However, approximation (58) is more efficient, it greatly simplifies the procedure for the self-consistent calculations and gives slightly lower values for the critical angular velocity and the polar radius.

\section{NON-LINEAR EQUILIBRIUM EQUATION}

To find approximate solutions of the equilibrium equation at $n>1$ in the work [7], the linearization procedure was proposed, which was used later in all the works using analytical approach. As was shown in the previous sections, in the transition from the polytrope with index $n=0$ to the polytrope with $n=1$ the maximal value of angular velocity decreases approximately in 1.9 times. From the results of the works [8] - [12], the approximate relation $\Omega_{\max }(n) \approx 2^{1-n} \Omega_{\max }(1)$ follows, where $\Omega_{\max }(n)$ is the maximal value of angular velocity for the polytrope with index $n$. This is the basis for linearizing equation (9)

$$
Y_{n}(\xi, \theta)=\tilde{y}_{n}(\xi)+\Omega^{2} \Psi_{n}(\xi, \theta) .
$$

Selecting the function of zero order approximation $\tilde{y}_{n}(\xi)$ in the form of the Emden function $y_{n}(\xi)$ (as in the works $[7,8])$, which is the solution of equation (27), is not optimal for the following reasons. The functions $y_{n}(\xi)$ are alternating, and the solution of equation (9) $Y(\xi, \theta) \geq 0$ by definition. Since at this selection, the function $\Psi_{n}(\xi, \theta)$ satisfies the equation

$$
\Delta(\xi, \theta) \Psi_{n}(\xi, \theta)=1-n \cdot y_{n}^{n-1}(\xi) \Psi_{n}(\xi, \theta),
$$

then for non-integer values the polytropic index $n$ has complex solutions. It should be noted that with integer values of the polytropic index $n$ in the role of zero approximation, we can use the function $y_{n}(\xi)$. This leads to small errors, since the density of matter in the surface layers is small.

In order to obtain a self-consistent solution of the problem, we select the function $\tilde{y}_{n}(\xi)$, which satisfies the equation

$$
\Delta(\xi) \tilde{y}_{n}(\xi)=G_{n}^{2}-\tilde{y}^{n}(\xi)
$$

Here $G_{n}$ is an auxiliary parameter; this is such a value of angular velocity, at which the solution of equation (73) does not accept negative values, and in the vicinity of some point $\tilde{\xi}_{1}(n)$ the function $\tilde{y}_{n}(\xi)$ reaches the minimum, moreover $\tilde{y}_{n}\left(\tilde{\xi}_{1}(n)\right)=0$. The solutions of equation (73) for several values of the polytropic index $n$ are shown in Fig. 8.

The corresponding values of parameter $G_{n}$ are given in Table 4 . It is easy to see that these values are close to the maximum value of angular velocity at fixed polytropic indexes. For further calculations, the functions $\tilde{y}_{n}(\xi)$ are used in the range $0 \leq \xi \leq \tilde{\xi}_{1}(n)$.

\begin{tabular}{|c|c|c|c|c|}
\hline$n$ & 1.5 & 2.0 & 2.5 & 3.0 \\
\hline$G_{n}$ & 0.287319 & 0.194602 & 0.128602 & 0.0808936 \\
\hline
\end{tabular}

Table 4 . Dependence of the parameter $G_{n}$ on the polytropic index $n$

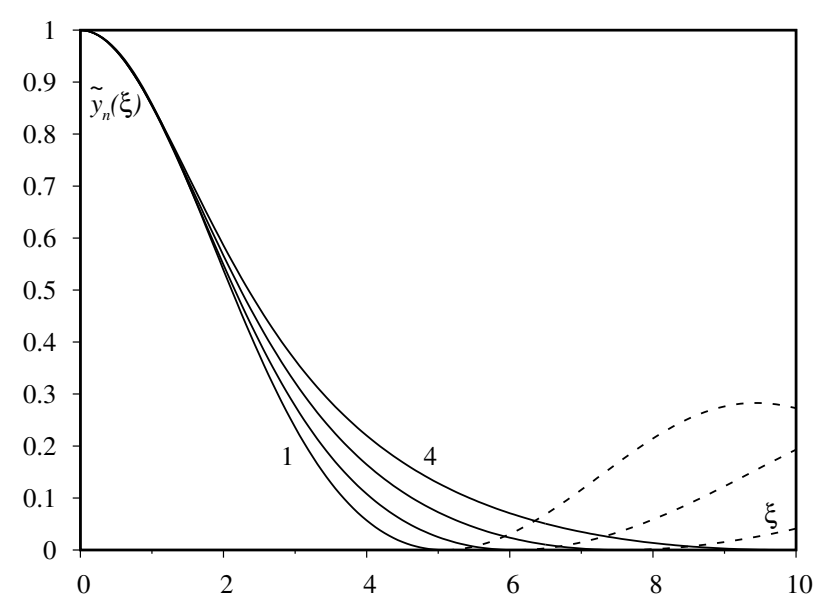

Fig. 8. The solutions of equation (73) for several values of the polytropic index $1.5 \leq n \leq 3.0, \Delta n=0.5$. Curve 1 corresponds to the polytropic index $n=1.5$, curve $4-$ $n=3.0$

The equation for the function $\Psi_{n}(\xi, \theta)$ now takes the form

$$
\Omega^{2} \Delta(\xi, \theta) \Psi_{n}(\xi, \theta)=\left(\Omega^{2}-G_{n}^{2}\right)-\Omega^{2} n \tilde{y}_{n}^{n-1}(\xi) \Psi_{n}(\xi, \theta) .
$$

By analogy to equation (59), we use the substitution

$$
\Psi_{n}(\xi, \theta)=\psi_{n, 0}(\xi \mid \Omega)+\sum_{l=1} \alpha_{2 l} P_{2 l}(t) \psi_{n, 2 l}(\xi)
$$

where $\alpha_{2 l}$ are the integration constants, $\psi_{n, 0}(\xi \mid \Omega)$ and $\psi_{n, 2 l}(\xi)$ are the functions of variable $\xi$. They are the solutions of the following linear equations

$$
\begin{aligned}
& \Omega^{2} \Delta_{\xi} \psi_{n, 0}(\xi \mid \Omega)=\left(\Omega^{2}-G_{n}^{2}\right)-\Omega^{2} n \tilde{y}_{n}^{n-1}(\xi) \psi_{n, 0}(\xi \mid \Omega), \\
& \Delta_{\xi} \psi_{n, 2 l}(\xi)=\left\{2 l(2 l+1) \xi^{-2}-n \tilde{y}_{n}^{n-1}(\xi)\right\} \psi_{n, 2 l}(\xi) .
\end{aligned}
$$

which correspond to the zero boundary conditions:

$$
\begin{aligned}
& \psi_{n, 0}(0 \mid \Omega)=\psi_{n, 2 l}(0)=0, \\
& \partial \psi_{n, 0}(\xi \mid \Omega) / \partial \xi=\partial \psi_{n, 2 l}(\xi) / \partial \xi=0 \text { at } \quad \xi \rightarrow 0 .
\end{aligned}
$$

The solutions of equations $\psi_{n, 0}(\xi \mid \Omega)$ and $\psi_{n, 2 l}(\xi)$ at $1 \leq$ $l \leq 3$ were found numerically. The functions $\psi_{n, 2 l}(\xi)$ are the analogous of Bessel's functions $j_{2 l}(\xi)$. 
Let us consider the definition of integration constants $\alpha_{2 l}$, using the integral form of the equilibrium equation.
Analogue (61) takes the following form:

$$
\begin{aligned}
& \tilde{y}_{n}(\xi)+\Omega^{2} \psi_{n, 0}(\xi \mid \Omega)+\Omega^{2} \sum_{l=1}^{\infty} \alpha_{2 l} \psi_{n, 2 l}(\xi) P_{2 l}(t) \\
& =1+\frac{\Omega^{2} \xi^{2}}{6}\left(1-P_{2}(t)\right)+\frac{1}{4 \pi} \int d \boldsymbol{\xi}^{\prime} Q\left(\boldsymbol{\xi}, \boldsymbol{\xi}^{\prime}\right) \\
& \times\left\{\tilde{y}_{n}^{n}\left(\xi^{\prime}\right)+n \tilde{y}_{n}^{n-1}\left(\xi^{\prime}\right) \Omega^{2}\left[\psi_{n, 0}\left(\xi^{\prime} \mid \Omega\right)+\sum_{l=1}^{\infty} \alpha_{2 l} \psi_{n, 2 l}\left(\xi^{\prime}\right) P_{2 l}\left(t^{\prime}\right)\right]\right\} .
\end{aligned}
$$

This equation can be simplified, taking into account the equation for $\tilde{y}_{n}(\xi)$ and $\psi_{n, 0}(\xi \mid \Omega)$ in the integral form:

$$
\begin{aligned}
& \tilde{y}_{n}(\xi)=1+\int_{0}^{\xi} d \xi^{\prime}\left[\frac{\left(\xi^{\prime}\right)^{2}}{\xi}-\xi^{\prime}\right]\left\{\tilde{y}_{n}^{n}\left(\xi^{\prime}\right)-G_{n}^{2}\right\}, \\
& \Omega^{2} \psi_{n, 0}(\xi \mid \Omega)=\int_{0}^{\xi} d \xi^{\prime}\left[\frac{\left(\xi^{\prime}\right)^{2}}{\xi}-\xi^{\prime}\right]\left\{\Omega^{2} \psi_{n, 0}\left(\xi^{\prime} \mid \Omega\right) n \tilde{y}_{n}^{n-1}\left(\xi^{\prime}\right)+G_{n}^{2}-\Omega^{2}\right\} .
\end{aligned}
$$

As a result, $\tilde{y}_{n}(\xi), \psi_{n, 0}(\xi \mid \Omega)$ is excluded from equation (78) and we obtain an analog of equation (61), namely

$$
\begin{aligned}
& \sum_{l=1}^{\infty} \alpha_{2 l} P_{2 l}(t) \psi_{n, 2 l}(\xi)=-\frac{\xi^{2}}{6} P_{2}(t)\{1+3 I(e)\} \\
& +\frac{1}{4 \pi} \int Q\left(\boldsymbol{\xi}, \boldsymbol{\xi}^{\prime}\right) \sum_{l=1}^{\infty} \alpha_{2 l} P_{2 l}\left(t^{\prime}\right) n \tilde{y}_{n}^{n-1}\left(\xi^{\prime}\right) \psi_{n, 2 l}\left(\xi^{\prime}\right) d \boldsymbol{\xi}^{\prime}
\end{aligned}
$$

Expanding the kernel $Q\left(\boldsymbol{\xi}, \boldsymbol{\xi}^{\prime}\right)$ in a series of the Legendre polynomials, integrating over the variable $\boldsymbol{\xi}^{\prime}$ in the vicinity of the rotational ellipsoid with eccentricity $e$ and equatorial radius $\xi_{e}$, equating the coefficients at the same factors $\xi^{2 l} P_{2 l}(t)$ in left and right sides of equation (78), we obtain a system of linear equations for the integration constants:

$$
\begin{aligned}
& \alpha_{2} S_{2,2}+\alpha_{4} S_{2,4}+\alpha_{6} S_{2,6}+\ldots=-\frac{1}{6}[1+3 I(e)] \\
& \alpha_{2} S_{4,2}+\alpha_{4} S_{4,4}+\alpha_{6} S_{4,6}+\ldots=0 \\
& \alpha_{2} S_{6,2}+\alpha_{4} S_{6,4}+\alpha_{6} S_{6,6}+\ldots=0
\end{aligned}
$$

The coefficients of this system are determined by the following expressions:

$$
\begin{aligned}
S_{2 l, 2 l} & =\int_{0}^{1} P_{2 l}^{2}(t) d t \xi_{0}^{-2 l}(t)\left\{(2 l+1) \psi_{n, 2 l}\left(\xi_{0}(t)\right)+\xi_{0}(t) \frac{d}{d \xi_{0}} \psi_{n, 2 l}\left(\xi_{0}(t)\right)\right\} ; \\
S_{2,2 l} & =-\int_{0}^{1} P_{2}(t) P_{2 l}(t) d t \int_{\xi_{1}(n)}^{\xi_{0}(t)} n \tilde{y}_{n}^{n-1}\left(\xi^{\prime}\right) \psi_{n, 2 l}\left(\xi^{\prime}\right)\left(\xi^{\prime}\right)^{-1} d \xi^{\prime} ; \quad l=2 ; 3 ; \\
S_{4,2 l} & =-\int_{0}^{1} P_{4}(t) P_{2 l}(t) d t \int_{\xi_{1}(n)}^{\xi_{0}(t)} n \tilde{y}_{n}^{n-1}\left(\xi^{\prime}\right) \psi_{n, 2 l}\left(\xi^{\prime}\right)\left(\xi^{\prime}\right)^{-3} d \xi^{\prime} ; \quad l=1 ; 3 ; \\
S_{6,2 l}= & -\int_{0}^{1} P_{6}(t) P_{2 l}(t) d t \int_{\xi_{1}(n)} n \tilde{y}_{n}^{n-1}\left(\xi^{\prime}\right) \psi_{n, 2 l}\left(\xi^{\prime}\right)\left(\xi^{\prime}\right)^{-5} d \xi^{\prime} ; \quad l=1 ; 2,
\end{aligned}
$$

and calculated numerically. 
The approximation of E. Milne-S. Chandrasekhar [7, 8] corresponds to

$$
\tilde{\alpha}_{2} \simeq-\frac{5}{6} \xi_{1}^{2}\left\{3 \psi_{n, 2}\left(\xi_{1}\right)+\xi_{1} \frac{d}{d \xi_{1}} \psi_{n, 2}\left(\xi_{1}\right)\right\}^{-1}
$$

where $\xi_{1} \equiv \xi_{1}(n)$ is the dimensionless radius of Emden's sphere for the polytropic index $n$. The selfconsistent calculation of rotational ellipsoid parameters is performed in the same way as at $n=1$, using the iteration method. In zero approximation $\alpha_{2}=\tilde{\alpha}_{2}, \alpha_{4}=$ $\alpha_{6}=0$.

Tables 5-8 showed the results of self-consistent calculations of the dependence of geometrical parameters of the polytropic stars, their masses and moments of inertia on the axis of rotation, as well as the dependence of integration constants $\alpha_{2 l}$ on the rotation velocity for the polytropic index $1.5 \leq n \leq 3.0$ at $\Delta n=0.5$. This range of the polytropic index is the most important from the application point of view, because it corresponds to degenerate dwarfs.

The dependence of angular velocity on equatorial and polar radii in different approximations for the polytropic indexes $n=1.5 ; 3.0$ was illustrated in Figs. 9-12. Curves 1 correspond to the approximation of E. MilneS. Chandrasekhar, curves 2 are built on the results of our calculations according to Tabs. 5, 8, curves $3-$ according to the work [10], curves 4 - according to the work [11]. In Fig. 12, curve 1 (the approximation of E. Milne-S. Chandrasekhar) almost coincides with R. James's results (curve 3), so we do not show it.

As can be seen from the Tables, the maximal value of angular rotational velocity decreases with an increase in $n$ proportional to $\Omega_{\max }(n) \approx 2^{1-n} \Omega_{\max }(1)$. Therefore, in the case of $n>1$ the approximate consideration of equilibrium becomes possible by linearizing of equation (9). In this case, the convergence of the series for the functions $\psi_{n, 2 l}(\xi) P_{2 l}(t)$ improves with an increase in the polytropic index, as evidenced by the results, which were shown in Tables $5-8$.

\begin{tabular}{|c|c|c|c|c|c|c|c|c|}
\hline$\Omega$ & $e(\Omega)$ & $\xi_{p}(\Omega)$ & $\xi_{e}(\Omega)$ & $\alpha_{2}(\Omega)$ & $\alpha_{4}(\Omega)$ & $\alpha_{6}(\Omega)$ & $\eta(n, \Omega)$ & $\zeta(n, \Omega)$ \\
\hline 0.01000 & 0.03727 & 3.65366 & 3.65620 & -11.38260 & 0.00609602 & $2.19394 \cdot 10^{-5}$ & 1.00266 & 1.00659 \\
\hline 0.02000 & 0.07465 & 3.65043 & 3.66064 & -11.40420 & 0.0245327 & $6.75745 \cdot 10^{-5}$ & 1.00351 & 1.00925 \\
\hline 0.03000 & 0.11199 & 3.64504 & 3.66812 & -11.44030 & 0.0555408 & 0.000352768 & 1.00493 & 1.01371 \\
\hline 0.04000 & 0.14939 & 3.63745 & 3.67874 & -11.49140 & 0.0996388 & 0.00125495 & 1.00694 & 1.02005 \\
\hline 0.05000 & 0.18688 & 3.62763 & 3.69268 & -11.55790 & 0.157522 & 0.00309018 & 1.00956 & 1.02836 \\
\hline 0.06000 & 0.22445 & 3.61552 & 3.71017 & -11.64060 & 0.230143 & 0.0066128 & 1.01283 & 1.03878 \\
\hline 0.07000 & 0.26216 & 3.60106 & 3.73153 & -11.74030 & 0.318724 & 0.0124912 & 1.01677 & 1.05147 \\
\hline 0.08000 & 0.30000 & 3.58416 & 3.75717 & -11.85810 & 0.424816 & 0.0219074 & 1.02145 & 1.06668 \\
\hline 0.09000 & 0.33805 & 3.56475 & 3.78762 & -11.99570 & 0.550374 & 0.0361455 & 1.02692 & 1.08468 \\
\hline 0.10000 & 0.37633 & 3.54271 & 3.82360 & -12.15480 & 0.697832 & 0.0570846 & 1.03327 & 1.10585 \\
\hline 0.11000 & 0.41493 & 3.51791 & 3.86607 & -12.33810 & 0.870274 & 0.0871608 & 1.04060 & 1.13069 \\
\hline 0.12000 & 0.45394 & 3.49016 & 3.91638 & -12.54870 & 1.07161 & 0.129713 & 1.04906 & 1.15985 \\
\hline 0.13000 & 0.49353 & 3.45924 & 3.97646 & -12.79150 & 1.30696 & 0.189748 & 1.05882 & 1.19422 \\
\hline 0.14000 & 0.53395 & 3.42479 & 4.04927 & -13.07320 & 1.58324 & 0.274923 & 1.07014 & 1.23502 \\
\hline 0.15000 & 0.57561 & 3.38626 & 4.13968 & -13.40430 & 1.91028 & 0.398865 & 1.08337 & 1.28410 \\
\hline 0.16000 & 0.61931 & 3.34263 & 4.25671 & -13.80320 & 2.30349 & 0.588075 & 1.09905 & 1.34434 \\
\hline 0.17000 & 0.66686 & 3.29167 & 4.42075 & -14.30850 & 2.79123 & 0.905728 & 1.11812 & 1.42093 \\
\hline 0.18000 & 0.72442 & 3.22687 & 4.70238 & -15.04000 & 3.44931 & 1.55731 & 1.14267 & 1.52634 \\
\hline 0.18100 & 0.73159 & 3.21888 & 4.74698 & -15.14150 & 3.53342 & 1.66473 & 1.14564 & 1.53974 \\
\hline 0.18200 & 0.73933 & 3.21040 & 4.79833 & -15.25400 & 3.62382 & 1.78653 & 1.14876 & 1.55405 \\
\hline 0.18300 & 0.74791 & 3.20128 & 4.85947 & -15.38200 & 3.72279 & 1.92639 & 1.15208 & 1.56952 \\
\hline 0.18400 & 0.75788 & 3.19128 & 4.93676 & -15.53560 & 3.83491 & 2.08953 & 1.15568 & 1.58670 \\
\hline 0.18410 & 0.75900 & 3.19022 & 4.94587 & -15.55310 & 3.8472 & 2.10738 & 1.15606 & 1.58855 \\
\hline 0.18420 & 0.76015 & 3.18914 & 4.95531 & -15.57120 & 3.85975 & 2.12554 & \begin{tabular}{|l|l|}
1.15644 \\
\end{tabular} & 1.59043 \\
\hline 0.18430 & 0.76133 & 3.18805 & 4.96514 & -15.58990 & 3.87258 & 2.14401 & 1.15684 & 1.59234 \\
\hline 0.18440 & 0.76255 & 3.18693 & 4.97537 & -15.60910 & 3.88571 & 2.16279 & 1.15724 & 1.59429 \\
\hline 0.18450 & 0.76380 & 3.18581 & 4.98606 & -15.62910 & 3.89917 & 2.18191 & 1.15764 & 1.59628 \\
\hline 0.18460 & 0.76511 & 3.18466 & 4.99726 & -15.65000 & 3.91302 & 2.20139 & 1.15805 & 1.59832 \\
\hline 0.18470 & 0.76647 & 3.18350 & 5.00908 & -15.67180 & 3.92732 & 2.22114 & 1.15847 & 1.60041 \\
\hline 0.18480 & 0.76788 & 3.18231 & 5.02154 & -15.69450 & 3.94204 & 2.24121 & 1.15890 & 1.60254 \\
\hline 0.18490 & 0.76938 & 3.18110 & 5.03489 & -15.71880 & 3.95747 & 2.26163 & 1.15934 & 1.60476 \\
\hline 0.18491 & 0.76953 & 3.18097 & 5.03626 & -15.72130 & 3.95903 & 2.26368 & 1.15939 & 1.60498 \\
\hline 0.18492 & 0.76968 & 3.18085 & 5.03764 & -15.72370 & 3.96058 & 2.26574 & 1.15943 & 1.60521 \\
\hline 0.18493 & 0.76983 & 3.18073 & 5.03902 & -15.72620 & 3.96215 & 2.26782 & 1.15948 & 1.60543 \\
\hline 0.18494 & 0.76999 & 3.18060 & 5.04046 & -15.72880 & 3.96378 & 2.2699 & 1.15952 & 1.60566 \\
\hline
\end{tabular}

Table 5. Dependence of the model characteristics with index $n=1.5$ on angular velocity 


\begin{tabular}{|c|c|c|c|c|c|c|c|c|}
\hline$\Omega$ & $e(\Omega)$ & $\xi_{p}(\Omega)$ & $\xi_{e}(\Omega)$ & $\alpha_{2}(\Omega)$ & $\alpha_{4}(\Omega)$ & $\alpha_{6}(\Omega)$ & $\eta(n, \Omega)$ & $\zeta(n, \Omega)$ \\
\hline 0.01000 & 0.04721 & 4.34935 & 4.35421 & -11.76340 & 0.00246147 & $1.48767 \cdot 10^{-5}$ & 1.00171 & 1.00488 \\
\hline 0.02000 & 0.09451 & 4.34402 & 4.36354 & -11.79790 & 0.00989875 & 0.00012479 & 1.00277 & 1.00839 \\
\hline 0.03000 & 0.14201 & 4.33505 & 4.37943 & -11.85630 & 0.0224803 & 0.00063061 & 1.00456 & 1.01432 \\
\hline 0.04000 & 0.18980 & 4.32233 & 4.40235 & -11.93980 & 0.04049 & 0.00203777 & 1.00710 & 1.02282 \\
\hline 0.05000 & 0.23799 & 4.30569 & 4.43305 & -12.05020 & 0.0643543 & 0.00511351 & 1.01043 & 1.03407 \\
\hline 0.06000 & 0.28674 & 4.28492 & 4.47267 & -12.19020 & 0.0946777 & 0.0110026 & 1.01460 & 1.04837 \\
\hline 0.07000 & 0.33622 & 4.25972 & 4.52288 & -12.36350 & 0.132292 & 0.0213357 & 1.01970 & 1.06613 \\
\hline 0.08000 & 0.38668 & 4.22972 & 4.58614 & -12.57560 & 0.17834 & 0.0385013 & 1.02583 & 1.08790 \\
\hline 0.09000 & 0.43851 & 4.19441 & 4.66638 & -12.83470 & 0.234427 & 0.0660378 & 1.03312 & 1.11449 \\
\hline 0.10000 & 0.49235 & 4.15304 & 4.77023 & -13.15390 & 0.302915 & 0.109525 & 1.04180 & 1.14709 \\
\hline 0.11000 & 0.54939 & 4.10427 & 4.91037 & -13.55680 & 0.387596 & 0.17879 & 1.05218 & 1.18763 \\
\hline 0.12000 & 0.61252 & 4.04496 & 5.11712 & -14.09670 & 0.49591 & 0.295119 & 1.06482 & 1.23955 \\
\hline 0.13000 & 0.69297 & 3.96298 & 5.51494 & -14.97470 & 0.652591 & 0.533935 & 1.08102 & 1.31176 \\
\hline 0.13100 & 0.70393 & 3.95164 & 5.58787 & -15.11580 & 0.674935 & 0.575974 & 1.08299 & 1.32119 \\
\hline 0.13200 & 0.71647 & 3.93889 & 5.67916 & -15.28500 & 0.700528 & 0.626144 & 1.08508 & 1.33147 \\
\hline 0.13300 & 0.73205 & 3.92384 & 5.80605 & -15.50820 & 0.732067 & 0.689267 & 1.08738 & 1.34318 \\
\hline 0.13400 & 0.76146 & 3.90235 & 6.09263 & -15.97620 & 0.787996 & 0.783569 & 1.09038 & 1.36011 \\
\hline 0.13401 & 0.76245 & 3.90193 & 6.10328 & -15.99310 & 0.789697 & 0.785217 & 1.09045 & 1.36051 \\
\hline 0.13402 & 0.76370 & 3.90144 & 6.11673 & -16.01470 & 0.791813 & 0.78704 & 1.09052 & 1.36101 \\
\hline 0.13403 & 0.76558 & 3.90081 & 6.13717 & -16.04740 & 0.79495 & 0.789276 & 1.09062 & 1.36172 \\
\hline
\end{tabular}

Table 6. Dependence of the model characteristics with index $n=2.0$ on angular velocity

\begin{tabular}{|c|c|c|c|c|c|c|c|c|}
\hline$\Omega$ & $e(\Omega)$ & $\xi_{p}(\Omega)$ & $\xi_{e}(\Omega)$ & $\alpha_{2}(\Omega)$ & $\alpha_{4}(\Omega)$ & $\alpha_{6}(\Omega)$ & $\eta(n, \Omega)$ & $\zeta(n, \Omega)$ \\
\hline 0.01000 & 0.06322 & 5.35048 & 5.36121 & -11.58170 & 0.000809512 & $4.90288 \cdot 10^{-6}$ & 1.00099 & 1.00332 \\
\hline 0.02000 & 0.12681 & 5.34119 & 5.38467 & -11.64060 & 0.00325686 & $6.97289 \cdot 10^{-5}$ & 1.00236 & 1.00819 \\
\hline 0.03000 & 0.19122 & 5.32534 & 5.42545 & -11.74210 & 0.00740741 & 0.000363718 & 1.00468 & 1.01652 \\
\hline 0.04000 & 0.25696 & 5.30234 & 5.48653 & -11.89210 & 0.0133808 & 0.00119972 & 1.00800 & 1.02861 \\
\hline 0.05000 & 0.32473 & 5.27131 & 5.57316 & -12.10030 & 0.0213781 & 0.00310439 & 1.01239 & 1.04498 \\
\hline 0.06000 & 0.39557 & 5.23087 & 5.69487 & -12.38390 & 0.0317358 & 0.0069595 & 1.01797 & 1.06648 \\
\hline 0.07000 & 0.47143 & 5.17877 & 5.87092 & -12.77590 & 0.0450651 & 0.014342 & 1.02495 & 1.09449 \\
\hline 0.08000 & 0.55692 & 5.10985 & 6.15057 & -13.35480 & 0.0627473 & 0.0286666 & 1.03366 & 1.13161 \\
\hline 0.09000 & 0.67342 & 4.99964 & 6.78465 & -14.47900 & 0.0910807 & 0.0638903 & 1.04496 & 1.18523 \\
\hline 0.09100 & 0.69208 & 4.98073 & 6.93480 & -14.70970 & 0.0960785 & 0.071603 & 1.04635 & 1.19268 \\
\hline 0.09200 & 0.71796 & 4.95578 & 7.17953 & -15.06120 & 0.103147 & 0.0825558 & 1.04789 & 1.20150 \\
\hline 0.09210 & 0.72160 & 4.95260 & 7.21777 & -15.11380 & 0.104136 & 0.08401 & 1.04806 & 1.20256 \\
\hline 0.09220 & 0.72570 & 4.94919 & 7.26216 & -15.17420 & 0.105245 & 0.0855875 & 1.04824 & 1.20369 \\
\hline 0.09230 & 0.73055 & 4.94543 & 7.31635 & -15.24710 & 0.106543 & 0.0873363 & 1.04843 & 1.20493 \\
\hline 0.09240 & 0.73690 & 4.94109 & 7.39002 & -15.34500 & 0.108207 & 0.0893592 & 1.04865 & 1.20641 \\
\hline 0.09241 & 0.73769 & 4.94060 & 7.39946 & -15.35750 & 0.108411 & 0.0895861 & 1.04867 & 1.20658 \\
\hline 0.09242 & 0.73855 & 4.94010 & 7.40962 & -15.37090 & 0.10863 & 0.0898219 & 1.04869 & 1.20676 \\
\hline 0.09243 & 0.73947 & 4.93957 & 7.42058 & -15.38540 & 0.108862 & 0.0900652 & 1.04872 & 1.20694 \\
\hline 0.09244 & 0.74045 & 4.93902 & 7.43246 & -15.40100 & 0.109112 & 0.0903167 & 1.04875 & 1.20714 \\
\hline 0.09245 & 0.74156 & 4.93844 & 7.44583 & -15.41870 & 0.109389 & 0.0905842 & 1.04877 & 1.20736 \\
\hline 0.09246 & 0.74281 & 4.93782 & 7.46104 & -15.43880 & 0.109699 & 0.0908674 & 1.04880 & 1.20760 \\
\hline 0.09247 & 0.74431 & 4.93713 & 7.47942 & -15.46300 & 0.110068 & 0.0911784 & 1.04884 & 1.20787 \\
\hline
\end{tabular}

Table 7. Dependence of the model characteristics with index $n=2.5$ on angular velocity 


\begin{tabular}{|c|c|c|c|c|c|c|c|c|}
\hline$\Omega$ & $e(\Omega)$ & $\xi_{p}(\Omega)$ & $\xi_{e}(\Omega)$ & $\alpha_{2}(\Omega)$ & $\alpha_{4}(\Omega)$ & $\alpha_{6}(\Omega)$ & $\eta(n, \Omega)$ & $\zeta(n, \Omega)$ \\
\hline 0.01000 & 0.09282 & 6.89320 & 6.92308 & -11.58950 & 0.000242961 & $2.11767 \cdot 10^{-6}$ & 1.00077 & 1.00309 \\
\hline 0.02000 & 0.18743 & 6.87397 & 6.99798 & -11.71670 & 0.00097408 & $3.52237 \cdot 10^{-5}$ & 1.00262 & 1.01058 \\
\hline 0.03000 & 0.28608 & 6.83926 & 7.13747 & -11.95040 & 0.0022038 & 0.00018928 & 1.00577 & 1.02366 \\
\hline 0.04000 & 0.39242 & 6.78413 & 7.37500 & -12.33800 & 0.00397054 & 0.000658839 & 1.01035 & 1.04346 \\
\hline 0.05000 & 0.51510 & 6.69711 & 7.81106 & -13.01180 & 0.00643835 & 0.00189636 & 1.01660 & 1.07241 \\
\hline 0.05100 & 0.52911 & 6.68556 & 7.87645 & -13.10850 & 0.00674501 & 0.00210223 & 1.01734 & 1.07600 \\
\hline 0.05200 & 0.54366 & 6.67319 & 7.94870 & -13.21390 & 0.00706879 & 0.0023324 & 1.01809 & 1.07974 \\
\hline 0.05300 & 0.55885 & 6.65988 & 8.02932 & -13.32970 & 0.00741316 & 0.00259151 & 1.01888 & 1.08367 \\
\hline 0.05400 & 0.57483 & 6.64541 & 8.12050 & -13.45850 & 0.00778304 & 0.00288602 & 1.01968 & 1.08779 \\
\hline 0.05500 & 0.59181 & 6.62945 & 8.22539 & -13.60360 & 0.00818584 & 0.00322535 & 1.02052 & 1.09214 \\
\hline 0.05600 & 0.61014 & 6.61155 & 8.34909 & -13.77060 & 0.00863335 & 0.00362405 & 1.02138 & 1.09674 \\
\hline 0.05700 & 0.63040 & 6.59087 & 8.50054 & -13.96880 & 0.00914621 & 0.00410702 & 1.02228 & 1.10167 \\
\hline 0.05800 & 0.65375 & 6.56588 & 8.69815 & -14.21690 & 0.00976614 & 0.00472256 & 1.02322 & 1.10701 \\
\hline 0.05900 & 0.68340 & 6.53284 & 8.99392 & -14.56660 & 0.0106051 & 0.00559054 & 1.02422 & 1.11303 \\
\hline 0.05910 & 0.68705 & 6.52876 & 9.03453 & -14.61270 & 0.0107125 & 0.00570237 & 1.02432 & 1.11369 \\
\hline 0.05920 & 0.69092 & 6.52445 & 9.07869 & -14.66230 & 0.0108272 & 0.0058213 & 1.02443 & 1.11437 \\
\hline 0.05930 & 0.69506 & 6.51989 & 9.12724 & -14.71620 & 0.0109508 & 0.00594865 & 1.02453 & 1.11508 \\
\hline 0.05940 & 0.69954 & 6.51501 & 9.18140 & -14.77560 & 0.0110854 & 0.00608601 & 1.02464 & 1.11581 \\
\hline 0.05950 & 0.70448 & 6.50976 & 9.24315 & -14.84250 & 0.0112348 & 0.0062359 & 1.02475 & 1.11657 \\
\hline 0.05960 & 0.71008 & 6.50402 & 9.31583 & -14.92010 & 0.0114048 & 0.00640189 & 1.02487 & 1.11738 \\
\hline 0.05970 & 0.71678 & 6.49758 & 9.40638 & -15.01540 & 0.0116074 & 0.00659069 & 1.02499 & 1.11827 \\
\hline 0.05980 & 0.72582 & 6.48994 & 9.53517 & -15.14850 & 0.0118771 & 0.00681817 & 1.02512 & 1.11932 \\
\hline 0.05981 & 0.72700 & 6.48906 & 9.55256 & -15.16630 & 0.0119118 & 0.00684466 & 1.02513 & 1.11944 \\
\hline 0.05982 & 0.72828 & 6.48814 & 9.57151 & -15.18570 & 0.0119491 & 0.00687227 & 1.02515 & 1.11957 \\
\hline 0.05983 & 0.72969 & 6.48719 & 9.59249 & -15.20720 & 0.0119898 & 0.00690127 & 1.02516 & 1.11971 \\
\hline 0.05984 & 0.73127 & 6.48616 & 9.61632 & -15.23150 & 0.0120353 & 0.0069321 & 1.02518 & 1.11986 \\
\hline 0.05985 & 0.73313 & 6.48504 & 9.64454 & -15.26040 & 0.0120881 & 0.00696567 & 1.02519 & 1.12003 \\
\hline 0.05986 & 0.73550 & 6.48377 & 9.68074 & -15.29740 & 0.0121541 & 0.00700368 & 1.02521 & 1.12023 \\
\hline 0.05987 & 0.73961 & 6.48201 & 9.74434 & -15.36260 & 0.0122653 & 0.00705586 & 1.02524 & 1.12055 \\
\hline
\end{tabular}

Table 8. Dependence of the model characteristics with index $n=3.0$ on angular velocity

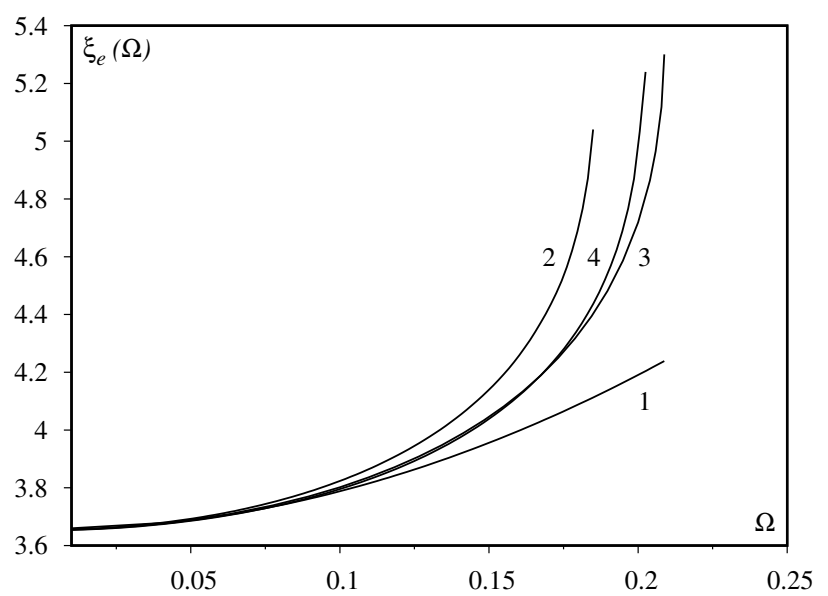

Fig. 9. Dependence of the equatorial radius $\xi_{e}(\Omega)$ on the rotation velocity $\Omega$ for the polytropic index $n=1.5$ in different approximations. Curve 1 is built on the results of the work [8], curve 2 is built according to Table 5 , curve 3 corresponds to the results [10], curve 4 corresponds to the results [11] 


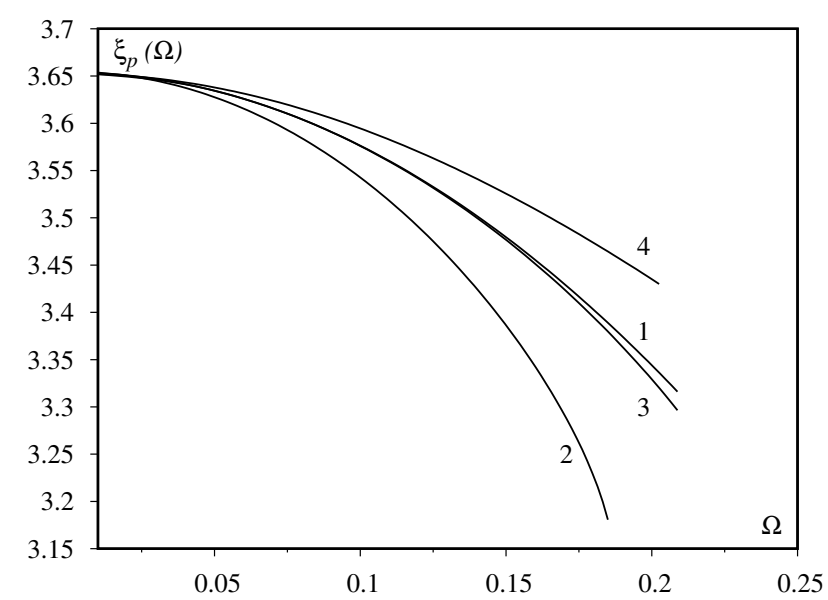

Fig. 10. Dependence of the polar radius $\xi_{p}(\Omega)$ on the rotation velocity $\Omega$ for the polytropic index $n=1.5$ in different approximations. Curve 1 is built on the results of the work [8], curve 2 is built according to Table 5 , curve 3 corresponds to the results [10], curve 4 corresponds to the results [11]

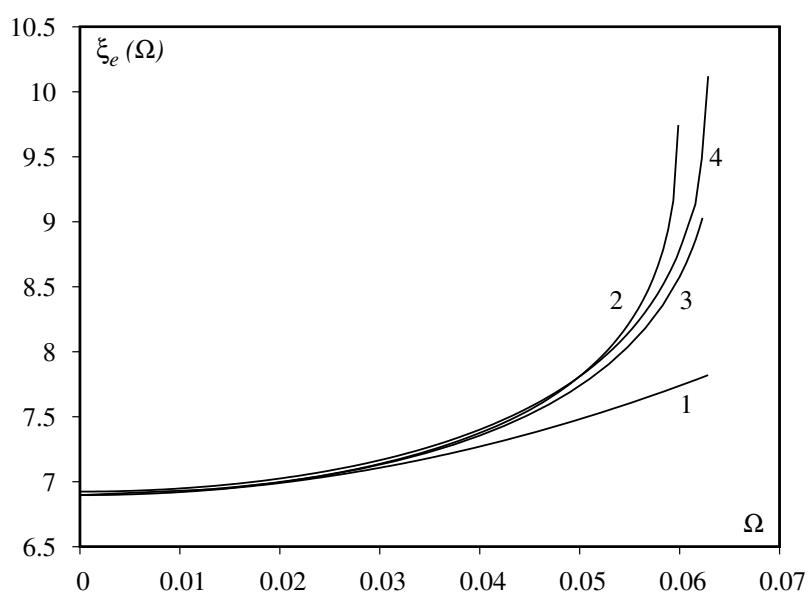

Fig. 11. Dependence of the equatorial radius $\xi_{e}(\Omega)$ on the rotation velocity $\Omega$ for the polytropic index $n=3.0$ in different approximations. Curve 1 is built on the results of the works [7,8], curve 2 is built according to Table 8 , curve 3 corresponds to the results [10], curve 4 corresponds to the results [11]

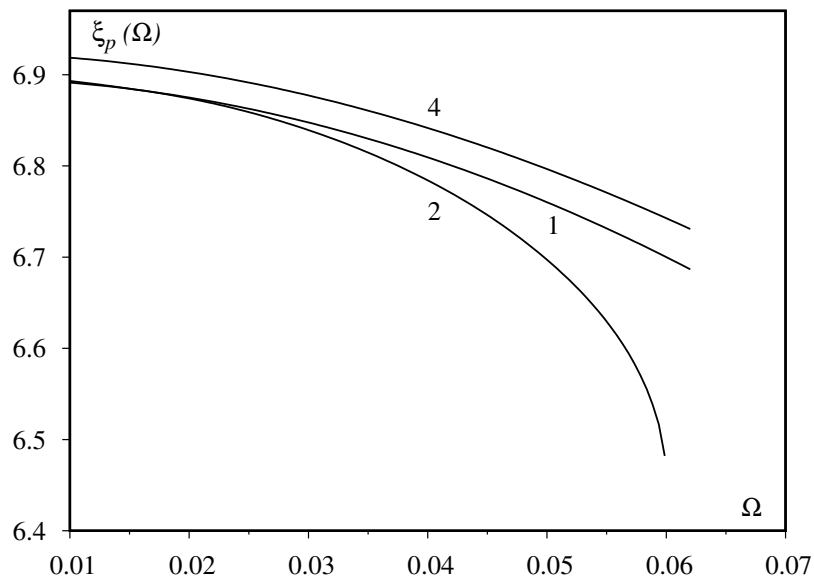

Fig. 12. Dependence of the polar radius $\xi_{p}(\Omega)$ on the rotation velocity $\Omega$ for the polytropic index $n=3.0$ in different approximations. Curve 1 is built on the results of the works $[7,8]$, curve 2 is built according to Table 8 , curve 4 corresponds to the results [11] 


\section{CONCLUSIONS}

Simultaneous employment of the differential and integral form of the mechanical equilibrium equation yields a closed system of expressions to determine the geometrical and physical characteristics of the polytropic star with axial rotation.

We have proposed two variants of the solution in the form of expansions for the Bessel functions and the Legendre polynomials for the polytrope with index $n=1$. The variant (58) is proven to be more effective: it has better convergence, which simplifies the procedure of finding the integration constants and gives a lower value of the angular velocity.

In the case of $n>1$, we used the standard linearization method of the equilibrium equation, which is known from the works $[7,8]$. The generalization of this method proposed by us consists in the usage the multicomponent trial functions and zero approximation, which is different from the Emden functions, and which improves the convergence of the series for the Legendre polynomials.

As was shown in Figs. 6, 7, the curves of the dependence of polar and equatorial radii, which are given by other authors, coincide at small values of $\Omega$ and are quite different near $\Omega_{\max }(n)$. The general pattern is as follows: as the calculations become more precise, the equatorial radius increases and the polar radius decreases. The critical polytropic characteristics $\Omega_{\max }(n), \xi_{e}\left(\Omega_{\max }(n)\right), \xi_{p}\left(\Omega_{\max }(n)\right)$ are the indicator of the precision of the calculations. Table 9 showed these parameters, which are obtained in the works [8, 10-12] as well as from our Tables 2 and $5-8$.

\begin{tabular}{|c||c|c|c|c|c|}
\hline$n$ & 1.0 & 1.5 & 2.0 & 2.5 & 3.0 \\
\hline$\Omega_{\max }^{C h}[8]$ & 0.3315 & - & 0.1805 & - & 0.0793 \\
\hline$\Omega_{\max }^{*}$ & 0.2447 & 0.18494 & 0.13403 & 0.09247 & 0.05987 \\
\hline$\Omega_{\max }^{J}[10]$ & 0.2891 & 0.2088 & 0.1466 & 0.0992 & 0.0623 \\
\hline$\Omega_{\max }^{M}[11]$ & 0.2755 & 0.2025 & 0.1411 & 0.0965 & 0.0628 \\
\hline$\Omega_{\max }^{C}[12]$ & 0.3264 & - & - & - & - \\
\hline \hline$\xi_{e}^{C h}$ & 3.91838 & - & 5.30746 & - & 8.36648 \\
$\xi_{p}^{C h}$ & 2.62374 & - & 3.91491 & - & 6.55314 \\
\hline$\xi_{e}^{*}$ & 4.40267 & 5.04046 & 6.13717 & 7.47942 & 9.74434 \\
$\xi_{p}^{*}$ & 2.61944 & 3.18060 & 3.90081 & 4.93713 & 6.48201 \\
\hline$\xi_{e}^{J}$ & 4.7349 & 5.3007 & 6.12214 & 7.40012 & 9.02964 \\
$\xi_{p}^{J}$ & 2.6942 & 3.2964 & 4.05671 & 5.10233 & 6.68477 \\
\hline$\xi_{e}^{M}$ & 4.54 & 5.24 & 6.33 & 7.72 & 10.12 \\
$\xi_{p}^{M}$ & 2.92 & 3.49 & 4.15 & 5.18 & 6.72 \\
\hline$\xi_{e}^{C}$ & 4.9973 & - & - & - & - \\
$\xi_{p}^{C}$ & 2.6534 & - & - & - & - \\
\hline$\xi_{E}(n)$ & 3.1416 & 3.6538 & 4.3529 & 5.3553 & 6.8969 \\
\hline
\end{tabular}

Table 9. Critical values of angular velocity, polar and equatorial radii ( $*$ denotes our results)

As was shown in Table, $\Omega_{\max }(n)$ calculated by us are the lowest, as well as $\xi_{p}\left(\Omega_{\max }\right)$. The data scattering of $\xi_{e}\left(\Omega_{\max }\right)$ in different authors is much larger than the data scattering of $\xi_{p}\left(\Omega_{\max }\right)$, which is caused by the approximations and errors of calculations. One of the objective indicators of the reliability of the calculations is the dependence of the values $\Delta \xi_{e} \equiv \xi_{e}\left(\Omega_{\max }(n)\right)-\xi_{E}(n)$ and $\Delta \xi_{p}(n) \equiv \xi_{E}(n)-\xi_{p}\left(\Omega_{\max }(n)\right)$ on the polytropic index, where $\xi_{E}(n)$ is the Emden radius. As was shown in Fig. 13, the value $\Delta \xi_{p}(n)$ is the monotonically decreasing function of the polytropic index $n$ both in the our work and in the works [8] and [10]. The value $\Delta \xi_{p}(n)$, which is calculated according to the work [11], does not have the monotonical dependence on the $n$, which is caused by negligence in the calculations of the fitting constants. Therefore, the results of the work [11] are less reliable than the results of other works. The value $\Delta \xi_{e}(n)$ is the monotonically increasing function of the polytropic index $n$ in all the works cited above (without taking into account the data of the work [10] for the $n=3$, which indicates the error).
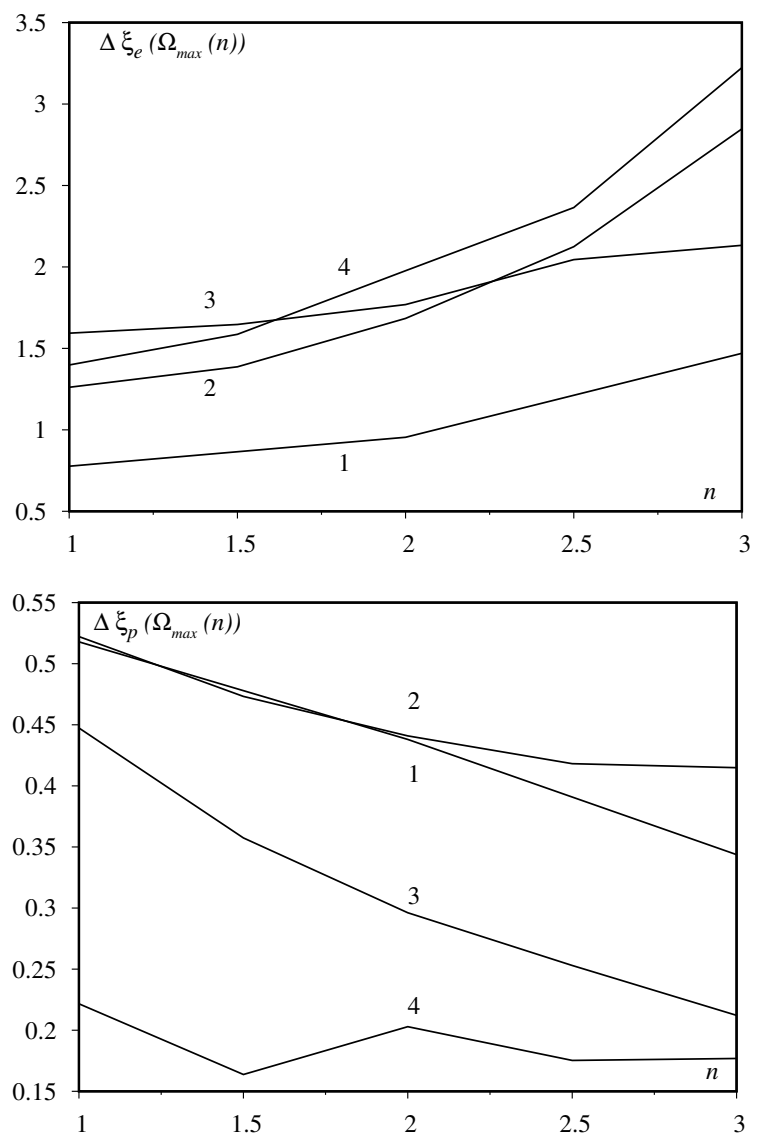

Fig. 13. Dependence of the values $\Delta \xi_{e} \equiv \xi_{e}\left(\Omega_{\max }(n)\right)-\xi_{E}(n)$ and $\Delta \xi_{p}(n) \equiv \xi_{E}(n)-\xi_{p}\left(\Omega_{\max }(n)\right)$ on the polytropic index in different approximations. Curves 1 are built on the results of the work [8], curves 2 are built according to the our results, curves 3 correspond to the results [10], curves 4 correspond to the results [11]

The works $[8,10,12]$ use too simple approximations for the calculations of the equilibrium equation, which can be represented by the formula from the work [11]:

$$
Y_{n}(\xi, \theta)=\sigma_{n}^{(0)}(\xi)+\Omega^{2} \sigma_{n}^{(1)}(\xi)+\Omega^{2} \sigma_{n}^{(2)}(\xi) P_{2}(t) .
$$

The approach proposed by us is based on the expansions 
for the orthogonal functions, which give more opportunities to find the solutions with sufficient precision.

The effectiveness of our approach can be judged from the inverse problem of the theory - determination of the polytrope parameters for the specific observable star. As an example, we consider the star $\alpha$ Eridani, which is described in the works [13, 14]. The work [13] gives the observed data for this star: its mass $M=9.7466 \cdot 10^{30} \mathrm{~kg}$, its equatorial radius $R_{e}=8.3520 \cdot 10^{9} \mathrm{~m}$, the ratio $\xi_{p} / \xi_{e}=2 / 3$, which corresponds to the eccentricity of the auxiliary ellipsoid $e=0.7454$. The polytropic model of the star has four parameters: $K, \rho_{c}, n, \omega$, which need to be determined. As in the works $[13,14]$, we use a polytrope with index $n=1$. The system of expressions

$$
\begin{aligned}
& R_{e}(1, \Omega)=\lambda_{1} \xi_{e}(\Omega), M(1, \Omega)=4 \pi^{2} \rho_{c} \lambda_{1}^{3} \eta(\Omega), \\
& K=2 \pi G \lambda_{1}^{2}, \Omega=\omega\left(2 \pi G \rho_{c}\right)^{-1 / 2}
\end{aligned}
$$

allows us to determine the parameters of the polytropic model, if $R_{e}(1, \Omega)$ is replaced with the observed equatorial radius $R_{e}$, and $M(1, \Omega)$ - with the observed mass $M$. According to Table 2, the eccentricity $e(\Omega)=$ 0.7454 corresponds to the dimensionless angular veloci- ty $\Omega=0.23655$ and the dimensionless equatorial radius $\xi_{e}(\Omega)=4.02993$. Therefore, the polytropic parameters are obtained as follows: $K=1.80112 \cdot 10^{9} \mathrm{~Pa} \mathrm{~m}^{6}(\mathrm{~kg})^{-2}$, $\rho_{c}=22.5881 \mathrm{~kg} \mathrm{~m}^{-3}, \omega=2.30214 \cdot 10^{-5} \mathrm{~s}^{-1}$. According to Table 3, this eccentricity corresponds to the dimensionless angular velocity $\Omega=0.24396$ and the dimensionless equatorial radius $\xi_{e}(\Omega)=4.12304$, the polytropic parameters are obtained as follows: $K=1.72069 \cdot 10^{9} \mathrm{~Pa} \mathrm{~m}^{6}(\mathrm{~kg})^{-2}, \rho_{c}=24.0449 \mathrm{~kg} \mathrm{~m}^{-3}$, $\omega=2.44963 \cdot 10^{-5} \mathrm{~s}^{-1}$. In the work [13], where only the exact integral representation of the equilibrium equation solution is obtained in the analytical form, and then numerical calculations are used, the value $K=$ $1.75 \cdot 10^{9} \mathrm{~Pa} \mathrm{~m}^{6}(\mathrm{~kg})^{-2}$ is obtained. Thus, we obtained that the value of the parameter $K$ is close to the result of the work [13], the approximation (58) yields the value of this parameter with an excess, and the expression (59) - with a shortage. The approximate solution of the equilibrium equation in the form $0.5\left(Y_{1}(\xi, \theta)+\tilde{Y}_{1}(\xi, \theta)\right)$ (where $Y_{1}(\xi, \theta)$ is determined by the expression $(58)$, and $\tilde{Y}_{1}(\xi, \theta)$ is determined by the expression (59)) corresponds to the value of the parameter $K$, which practically coincides with the result of the work [13].
[1] H. J. Lane, Am. J. Sci. 50, 57 (1870); https://doi.or g/10.2475/ajs.s2-50.148.57.

[2] R. Emden, Gaskugeln: Anwendungen der mechanischen Wärmetheorie auf kosmologische und meteorologische Probleme (Teubner, Leipzig, Berlin, 1907).

[3] R. H. Fowler, Mon. Not. Roy. Astron. Soc. 91, 63 (1930); https://doi.org/10.1093/mnras/91.1.63.

[4] A. S. Eddington, The Internal Constitution of the Stars (Cambridge University Press, Cambridge, 1926); https : //doi.org/10.1017/CB09780511600005.

[5] S. Chandrasekhar, Astrophys. J. 74, 81 (1931);https : //doi.org/10.1086/143324.

[6] S. Chandrasekhar, Ellipsoidal Figures of Equilibrium (Yale University Press, New Haven, 1969).

[7] E. A. Milne, Mon. Not. Roy. Astron. Soc. 83, 118 (1923); https://doi.org/10.1093/mnras/83.3.118.

[8] S. Chandrasekhar, E. A. Milne, Mon. Not. Roy. Astron. Soc. 93, 390 (1933); https://doi.org/10.1093/mnras/ 93.5.390.

[9] Z. Kopal, Z. Astrophys. 14, 135 (1937); https://doi. org/10.1002/bbpc. 19580620909 .

[10] R. A. James, Astrophys. J. 140, 552 (1964); https:// doi.org/10.1086/147949.

[11] J. J. Monaghan, I. W. Roxburgh, Mon. Not. Roy. Astron. Soc. 131, 13 (1965); https://doi.org/10.1093/mnras/ 131.1.13.
[12] R. Caimmi, Astrophys. Space Sci. 71, 415 (1980); https : //doi.org/10.1007/BF00639402.

[13] D. Kong, K. Zhang, G. Schubert, Mon. Not. Roy. Astron. Soc. 448, 456 (2015); https://doi.org/10.1093/mnra s/stu2759.

[14] J. Knopik, P. Mach, A. Odrzywołek, Mon. Not. Roy. Astron. Soc. 467, 4965 (2017); https://doi.org/10.1 $093 / \mathrm{mnras} / \mathrm{stx} 164$

[15] M. V. Vavrukh, S. V. Smerechynskyi, N. L. Tyshko, J. Phys. Stud. 14, 4901 (2010); https://doi.org/10.3 0970/jps. 14.4901.

[16] M. V. Vavrukh, N. L. Tyshko, D. V. Dzikovskyi, O. M. Stelmakh, Math. Model. Comput. 6, 153 (2019); https://doi.org/10.23939/mmc2019.02.153.

[17] S. Chandrasekhar, An Introduction to the Study of Stellar Structure (University of Chicago Press, Chicago, 1939).

[18] S. L. Shapiro, S. A. Teukolsky, Black Holes, White Dwarfs and Neutron Stars (Ithaca, New York, Cornell University, 1983); http://doi.org/10.1002/97835276 17661.

[19] M. Abramowitz, I. A. Stegun, Handbook of Mathematical Functions With Formulas, Graphs, and Mathematical Tables (Government Printing Office Washington, 1972).

[20] R. A. Lyttleton, The Stability of Rotating Liquid Masses (Cambridge University Press, Cambridge, 1953). 


\title{
НОВИЙ ПІДХІД У ТЕОРІЇ РІВНОВАГИ ЗІР З ОСЬОВИМ ОБЕРТАННЯМ
}

\author{
М. В. Ваврух, Н. Л. Тишко, Д. В. Дзіковський \\ Лъвівсъкий націоналъний університет імені Івана Франка, \\ кафедра астрофізики, вул. Кирила і Мефоділ, 8, 79005 Лъвів, Украӥна
}

Запропоновано новий варіант опису рівноважних конфігурацій зір у межах моделі з твердотільним осьовим обертанням і політропним рівнянням стану $P(\mathbf{r})=K(\rho(\mathbf{r}))^{1+1 / n}$. Крім параметра $K$, модель має ще три параметри: $\rho_{c}$ - густина в центрі зорі, $\omega$ - кутова швидкість, $n-$ індекс політропи. Метою роботи є знайти розподіл речовини за об'ємом зорі на основі рівняння рівноваги при заданих параметрах моделі. Ми одночасно використовуємо рівняння рівноваги як у диференційній, так і в інтегральній формах. Це замкнута система рівнянь, що не потребує додаткової інформації. За допомогою підстановки $\mathbf{r}=\boldsymbol{\xi} \lambda_{n}, \rho(\mathbf{r})=\rho_{c} Y^{n}(\xi, \theta)\left(\lambda_{n}^{2}=K(1+n)\left[4 \pi G \rho_{c}^{1-1 / n}\right]^{-1}\right)$ система рівнянь набуває безрозмірного вигляду й залежить від двох безрозмірних параметрів - індексу політропи $(0<n \leq 5)$ та безрозмірної швидкості $\Omega=\omega\left(2 \pi G \rho_{c}\right)^{-1 / 2}$, яка для більшості зір є малою величиною. Модель $3 n=0$ відповідає нестисливій рідині, для неї відомий розв'язок і залежність характеристик від $\Omega$ в зоні стійкості $\left(0 \leq \Omega \leq \Omega_{\max }(0)\right)$. Випадок $n=1$ відіграє роль еталона. Ми знайшли два еквівалентні зображення точного розв'язку у вигляді безмежних рядів за поліномами Лежандра i сферичними функціями Бесселя за $n=1$. На цій основі запропоновано два наближені вирази зі скінченною кількістю доданків. За допомогою інтегрального рівняння рівноваги одержано систему лінійних алгебраїчних рівнянь для знаходження коефіцієнтів розкладів, яка розв'язується чисельно, методом ітерацій. Наведено таблиці залежності екваторіального та полярного радіусів поверхні політропи, маси та моменту інерції від $\Omega$, а також межу стійкості відносно обертання $\Omega_{\max }(1)$. Для $n>1$ використовуємо теорію збурень стосовно впливу обертання. Запропоновано нову методику лінеаризації рівняння рівноваги. Розв'язки лінеаризованих рівнянь зображені у вигляді скінченних розкладів за поліномами Лежандра й деякими функціями радіальної змінної $\psi_{n, 2 l}(\xi)$, розрахованими чисельним методом. Коефіцієнти розкладів визначено за методикою, розробленою для $n=1$. Знайдено межі існування ділянок стабільності $\left(\Omega_{\max }(n) \approx 2^{1-n} \Omega_{\max }(1)\right)$, розраховано геометричні параметри політроп та їхні фізичні характеристики, а також коефіцієнти розкладів як функції $\Omega$ для індексів $n=1.5,2.0,2.5,3.0$. Виконано порівняння розрахованих геометричних параметрів поверхонь політропи з результатами інших авторів. Одержані результати можна використати для побудови моделей конкретних зір (визначення параметрів $K, \rho_{c}, \omega, n$ ) за відомими спостережуваними даними. Як приклад ми розрахували параметри $K, \rho_{c}$ i $\omega$ для зорі $\alpha$ Eridani, використовуючи відому масу й екваторіальний радіус. У двох варіантах наближеного розв'язку за $n=1$ отримано значення параметра $K$, близьке до визначеного в роботі [13], яка грунтується на чисельних розрахунках.

Ключові слова: зорі-політропи, неоднорідні еліпсоїди, осьове обертання, рівняння механічної рівноваги, стабільність зір. 